\title{
Pore-by-pore modeling, analysis, and prediction of two-phase flow in mixed-wet rocks
}

\author{
Sajjad Foroughi $\odot,{ }^{*}$ Branko Bijeljic, Qingyang Lin $\odot$, Ali Q. Raeini, and Martin J. Blunt $\odot$ \\ Department of Earth Science and Engineering, Imperial College London, London, SW7 2AZ, United Kingdom
}

(Received 11 May 2020; accepted 9 July 2020; published 5 August 2020)

\begin{abstract}
A pore-network model is an upscaled representation of the pore space and fluid displacement, which is used to simulate two-phase flow through porous media. We use the results of pore-scale imaging experiments to calibrate and validate our simulations, and specifically to find the pore-scale distribution of wettability. We employ energy balance to estimate an average, thermodynamic, contact angle in the model, which is used as the initial estimate of contact angle. We then adjust the contact angle of each pore to match the observed fluid configurations in the experiment as a nonlinear inverse problem. The proposed algorithm is implemented on two sets of steady state micro-computed-tomography experiments for water-wet and mixed-wet Bentheimer sandstone. As a result of the optimization, the pore-by-pore error between the model and experiment is decreased to less than that observed between repeat experiments on the same rock sample. After calibration and matching, the model predictions for capillary pressure and relative permeability are in good agreement with the experiments. The proposed algorithm leads to a distribution of contact angle around the thermodynamic contact angle. We show that the contact angle is spatially correlated over around 4 pore lengths, while larger pores tend to be more oil-wet. Using randomly assigned distributions of contact angle in the model results in poor predictions of relative permeability and capillary pressure, particularly for the mixed-wet case.
\end{abstract}

DOI: 10.1103/PhysRevE.102.023302

\section{INTRODUCTION}

Multiphase flow through porous media has a crucial importance in applications as diverse as groundwater resources [1-3], geothermal fields [4], chemical transport [5-7], gas diffusion in fuel cells [8], oil recovery [9], environmental remediation [10], and $\mathrm{CO}_{2}$ sequestration [11]. The most important macroscopic parameters that describe multiphase flow through porous media are capillary pressure $\left(P_{c}\right)$ and relative permeability $\left(k_{r}\right)[9,12]$. These parameters depend on the geometry of the porous media as well as the fluid and rock properties, of which the most important is wettability.

Recent advances in $\mathrm{x}$-ray imaging have made it possible to study the pore structure of rocks at micron-scale resolution, and the fluids within them, providing valuable insights into multiphase flow processes [13-15]. From these pore-scale images, numerical models can be constructed that attempt to reproduce the displacement processes observed experimentally and predict macroscopic properties, such as relative permeability and capillary pressure [14,16,17].

Despite the growing body of pore-scale experimental data, currently, modeling has been limited to comparing predicted capillary pressures and relative permeabilities to experimental measurements, most of which were performed without pore-scale imaging to observe the microscopic configuration of fluids [17-20]. However, validating the models using experimentally measured macroscopic properties without considering pore-scale behavior is uncertain. Bultreys et al. [21] attributed this uncertainty to four factors: uncertainty in the experimental properties used to inform the model since the

*Corresponding author: s.foroughi@imperial.ac.uk number of input data, such as the pore-by-pore variation in wettabilty, far exceeds the information in the output required to characterize relative permeability and capillary pressure $[22,23]$; physics that is not captured by the model or numerical errors [24]; different length scales of the model and experiment [25]; and uncertainties in the experiment itself [26,27].

One particular source of uncertainty and ambiguity in model predictions is the assignment of wettability, or contact angle, on a pore-by-pore basis [28]. Most modeling studies have attempted to calibrate multiphase flow in a few rock types based only on the macroscopic experimental data, which, as discussed above, is not reliable [22]. The link between pore-scale wettability and macroscopic properties has been quantified with an assessment of uncertainty [29], which emphasizes the importance of using the correct input wettability.

In recent years, several studies have sought to validate image-based models against experimental micro-computedtomography (micro-CT) results including pore scale fluid distribution and topology $[21,28,30-34]$. Akai et al. [30] simulated an unsteady-state waterflooding experiment on a mixedwet carbonate rock using the lattice Boltzmann method incorporating locally measured contact angles [35]. Pore-by-pore comparison showed a good agreement between model and experiment for fluid occupancy as a function of pore size. This work demonstrated that a distribution of contact angle (rather than a uniform value) needs to be used as a model input to characterize wettability; however, the contact angle distribution had to be adjusted to obtain a good match to experiment. Bultreys et al. [21,32] used an image-based pore network model to compare against steady-state waterflood measurements in water-wet Bentheimer sandstone. They proposed a workflow that is based on mapping the fluid distribution from 
an experimental image to the extracted network model. They obtained a fluid occupancy that agreed with experiment in more than $75 \%$ of the network. Furthermore, Raeini et al. [28] performed pore-by-pore comparisons for a generalized network model on repeated unsteady state $\mathrm{CO}_{2}$ waterflood experiments in sandstones and carbonates [26,27] and on steadystate waterflood Bentheimer sandstone experiments. They quantified the mismatch between the micro-CT experiment and the network model by defining the discrepancy in pore occupancy. In many cases the discrepancy in occupancy was similar to that seen in repeated experiments, but larger errors were seen for the carbonate samples studied. A constant contact angle, or a uniform distribution, randomly distributed in space, was assumed. It would appear that even for the waterwet cases studied, some correlation in contact angle, either spatially, or with pore size, is required to improve the agreement with experimental measurements: we will show that including these correlations is indeed necessary to match the filling order accurately. Moreover, another challenge is to have a model that can be predictive for a range of wettability states. These image-based modeling studies $[21,28,32]$ were all performed for water-wet samples. However, mixed-wet behavior is expected to have an even higher level of uncertainty $[9,36]$. Here, we will study both water-wet and mixed-wet cases. Also, the previous works tried to minimize the mismatch at the end of drainage or imbibition, while in this paper, we match throughout waterflooding and not just at the end point. Furthermore, we will perform a quantitative comparison between macroscopic measured and predicted capillary pressures and relative permeabilities, which was not done previously.

In a quasistatic pore network model, the pore filling order is controlled by capillary pressure that is a function of geometry and wettability. The geometry of the network is extracted from a dry image of the porous medium that typically has a size of the order of 1 billion voxels. Hence, it can be argued that the geometry is well characterized [37,38]. However, wettability characterization is much more uncertain: wettability is controlled by contact angle, which is not well constrained in most cases [28]. This poses a considerable challenge since in order to get correct filling order, the correct contact angle is required.

The question now is what the contact angle should be. Blunt et al. [39] have used conservation of energy to derive a thermodynamically consistent contact angle. This provides an average angle that correctly captures the value needed in a quasistatic model to reproduce the displacement observed experimentally. However, we require individual contact angle values for each element in the network. AlRatrout et al. [40] developed a procedure to determine geometric contact angles from three-dimensional images. Whereas this can be used to determine values on a pore-by-pore basis [40], it has been shown that using the geometric angle, which represents, in many cases, a hinging angle on a rough or altered-wettability surface, does not lead to accurate predictions of displacement [41]. While, as we show, the thermodynamic contact angle does provide a good average value, we need to find the distribution about this average which enables the model to predict the observed displacement sequence.

We propose a nonlinear optimization to solve this inverse problem. We have measured, from experiments, the fluid distribution during waterflooding. We find contact angles on a pore-by-pore basis that correctly capture the observed changes in surface energy during the displacement and the measured configuration of fluid phases. This results in a nonlinear optimization, where we adjust contact angles on a pore-by-pore basis to minimize the mismatch between measured and predicted pore occupancy.

Even for repeated experiments, it is not possible to obtain a perfect repeatability. Based on the analysis of Andrew et al. [26,27] and Raeini et al. [28] the discrepancy in pore occupancy between repeated experiments was between 6 and $18 \%$. Ling et al. [42] studied the reproducibility of multiphase fluid distributions in six replicate experiments in identical microfluidic devices. They observed significant differences in the pore-scale fluid configurations for imbibition experiments. They showed that numerical models, while they could reproduce the overall behavior, could not be expected to match the experiments on a pore-by-pore basis. Therefore, the objective is to decrease mismatch between pore network model and experiment below the uncertainty in repeat experiments.

The paper is organized as follows, In Sec. II pore network modeling and the micro-CT multiphase flow experiments are described. In Sec. III the optimization workflow developed to perform pore-by-pore comparison of micro-CT experimental measurements against the pore network model is presented. This methodology is implemented on the waterwet and mixed-wet steady-state experiments in Secs. IV A and IV B, respectively, together with comparisons between predicted and measured capillary pressures and relative permeabilities. In Sec. IV C we examine the correlation in contact angle in our calibrated models and demonstrate that ignoring this correlation leads to poor predictions of multiphase flow properties.

\section{MATERIALS AND METHODS}

\section{A. Imaging experiments}

The measurements analysed in this study are from two steady-state multiphase flow experiments [42,43]. Both cases studied waterflooding in small rock samples of Bentheimer sandstone. Initially the rock contained oil and a low initial water saturation. Then water and oil were injected through the rock in a sequence of increasing water fractional flows (the ratio of the water volumetric flow rate to the total rate of oil and water) from 0 to 1 . At each fractional flow, injection continued until a steady state was reached, which was found when the pressure difference measured across the sample stabilized. High-resolution imaging was used to observe the pore-scale configuration of the oil and water and to quantify saturation. From the pressure differential and the imposed fractional flow, relative permeability could be determined. Capillary pressure was also estimated from the curvature of the oil-water menisci in the images. The experiments were performed on a water-wet sample, called WW in this paper [42], and a sample that had been left in contact with crude oil before the experiment which we label mixed-wet, MW [43]. Further details on the experiments and image analysis can be 
TABLE I. A summary of the properties of the images taken in the water-wet, WW, and mixed-wet, MW, experiments [42,43].

\begin{tabular}{lcc}
\hline \hline & WW [42] & MW [43] \\
\hline Number of images & 7 & 8 \\
Number of voxels & $1000 \times 1000 \times 3000$ & $1600 \times 1600 \times 3000$ \\
Cross section & square & circle \\
Voxel size $[\mu \mathrm{m}]$ & 3.58 & 3.58 \\
Number of pores & 68,323 & 97,698 \\
Number of throats & 150,619 & 220,272 \\
Imaged-based porosity & 0.205 & 0.231 \\
Initial oil saturation & 0.856 & 0.885 \\
Residual oil saturation & 0.383 & 0.113 \\
\hline \hline
\end{tabular}

found in Refs. [42,43]: the experiments were performed under capillary-controlled conditions.

Table I provides a summary of the properties of the images together with the measured initial and residual oil saturation in the experiments. The images were segmented into pore and grain, and the pore space partitioned into oil and water, using commercial image analysis software.

\section{B. Pore network extraction and modeling}

The segmented images were then run through a maximal ball pore-network extraction code [44,45]. This provides a quantitative topological representation of the void space divided into pores and throats. Pores represent wider regions of the void space, while throats represent restrictions between pores. The algorithm first determines the distance of each voxel of the void space from the closest solid; the local maxima of this distance map determines the center of pores. The boundary between two pores at the narrowest region of the void space defines the throat surface. Then the local maximum of the distance map on this surface is considered as the throat center. Details of the algorithm can be found in Refs. [44,45].

Flow simulation on the extracted network is performed using the approach developed by Valvatne and Blunt [19]. The network elements pores and throats have circular, triangular or square cross sections. Angular cross sections allow wetting layers to reside in the corners, as well as layers of oil, in oil-wet pores and throats, to be present between water in the corners and water in the center. Displacement proceeds in order of capillary pressure, computed semianalytically, based on the geometry of an element and its contact angle, by snap-off, pistonlike advance and cooperative pore filling. Here we use the modification proposed by Bultreys et al. [32] to obtain the initial condition for waterflooding: fluid occupancy at the end of drainage, the beginning of the waterflood, is taken directly from the micro-CT image.

The geometric inputs into the model are all determined from segmented images of the pore and grain from the experiments: these were obtained from high-quality images of the dry rock before flooding [32]. These inputs are the inscribed radii of pores and throats, the elements that they connect to, and the shape of the cross section. We assume that these are accurately represented in the images and therefore they are not altered during the calibration process.
The key uncertainty in the characterization of displacement is the wettability, namely the contact angle to assign to each pore and throat in the model. In what follows, this input is adjusted so that the predicted displacement sequence matches that observed in the experiments. Once the contact angle is known, the threshold capillary pressures, which determine the order in which pores and throats are invaded by water, are determined.

\section{VALIDATION AND CALIBRATION}

\section{A. Pore-by-pore error analysis}

In this study, to analyze and compare the model results and experiments we use the procedure of Bultreys et al. [21] and Raeini et al. [28]. The extracted pore network is used as an image analysis tool that makes possible the mapping of the distribution of fluids in the images to the corresponding network model. A quantitative comparison of predicted and measured results requires the definition of a mismatch indicator. Here filling state, denoted by $\phi$, is used. Based on the extracted network an inscribed sphere could be associated with each network element (a pore or throat), $i$. From the experiments, we identify the fluid phase that occupies more than half of the voxels associated with that inscribed sphere: this assigns a filling state which can be compared with the phase in the center of each element in the network model: $\phi_{i}=1$ if oil occupies more than half of the voxels of the corresponding inscribed sphere, otherwise $\phi_{i}=0$ for water. The average water occupancy, $\psi$, is defined as follows:

$$
\psi=\frac{\sum_{i} V_{i}\left(1-\phi_{i}\right)}{\sum_{i} V_{i}},
$$

where the sum over $i$ is over all pores and all throats. $V_{i}$ represents the volume of pore/throat $i$. During network extraction, a chain of maximal balls is found with the largest radius at the pore center to the smallest at the center of the throat surface. We partition volume between pores and throats as follows [45]: the border between a pore and its connected throat is at distance $l_{i}$ away from pore center, which is defined as follows:

$$
l_{i}=l_{i}^{t}\left(1-\alpha \frac{r_{t}}{r_{i}}\right),
$$

where $l_{i}^{t}$ is Euclidean distance between the pore center and the throat center, $r_{i}$ and $r_{t}$ are pore and throat inscribed radii. $\alpha$ is a pore-throat segmentation coefficient. We use $\alpha=0.6$ as in previous work [45]. Voxels in the void space are then uniquely assigned to a pore or throat. While this appears arbitrary, this division provides good predictions of absolute and relative permeability in simple systems [45]. The volume, $V_{i}$, is calculated by counting the number of void voxels in each pore or throat. Note that the sum of all the pore volumes plus the sum of all the throat volumes is equal to the total volume of the pore space. $\psi=1$ corresponds to a completely water-saturated network, while, $\psi=0$ is where the centers of all the pores and throats are filled with oil. Note that since we only consider a binary occupancy, this is not the same as saturation. 
We also determine the saturation, $S_{w}$. Saturation is defined in the model using semianalytical calculations of the water volume in each element. In the experiment, saturation is computed by counting the number of water voxels, $n_{w}$, in the segmented image

$$
S_{w}=n_{w} / n_{t},
$$

where $n_{t}$ is the total number of void space voxels. Here we do not need to associate voxels in the experiment with individual pores or throats.

To quantify the discrepancy in the filling state between the network model and the experimental data, we define the mean absolute discrepancy [28]:

$$
M_{A D}^{j}=\frac{\sum_{i} V_{i}\left|\phi_{i}^{m, j}-\phi_{i}^{e x, j}\right|}{\sum_{i} V_{i}},
$$

where the sum is over all pores and all throats. Note that $j$ represents the order of images for different fractional flows, $f_{w}^{j} . \phi_{i}^{e x, j}$ denotes the mapped filling state of elements from the experimental image $j . \phi_{i}^{m, j}$ is the filling state of elements obtained from the model at $S_{w}^{j}$ which is the saturation corresponding to image $j$. For each element (pore or throat) the absolute difference between experiment (ex) and the model (m) is zero when the predicted filling state of an element is the same as the experiment: otherwise the difference is equal to 1 . Here, we do not simply count the filling state discrepancy, which could be dominated by predictions in filling the smallest elements, but use a volume-weighted average. As water always fills the smallest elements in a water-wet medium, the frequency of correct filling states is quite high. However, these elements contribute little to flow so a more stringent metric is needed. The volume-weighted average, Eq. (4), is a more challenging indicator to match.

One subtlety is the stage in the model simulation at which we compare with the experiment. To make predictions of macroscopic properties, such as relative permeability and capillary pressure, which are traditionally presented as functions of saturation, we also need to match saturation. Hence we compare the model and the experiment at the point where the two saturations are equal. When we then optimize the filling sequence, we force the correct pore-by-pore occupancy at the same saturation. The overall occupancy $\psi$, may be different for the model and simulation: in what follows we define $\psi$ based on the experiments.

We can also define an integral measure to capture the discrepancy throughout a displacement:

$$
A_{M}\left(\psi_{f}\right)=\sum_{j=1}^{n_{f w}} \frac{\left(M_{A D}^{j}+M_{A D}^{j-1}\right)}{2}\left(\psi_{j}-\psi_{j-1}\right),
$$

where $\psi_{f}$, Eq. (1), is the volume-weighted fraction of waterfilled elements in the experiment at the end of the displacement and $n_{f_{w}}$ is the number of fractional flows considered after the initial condition. $j=0$ represents the initial condition. $M_{A D}^{0}=0$ since we input the initial filling states of elements, $\phi_{i}$ into the model. Equation (5) simply represents the trapezoid rule for calculating the area bounded by $M_{A D}$ for a finite series of images.

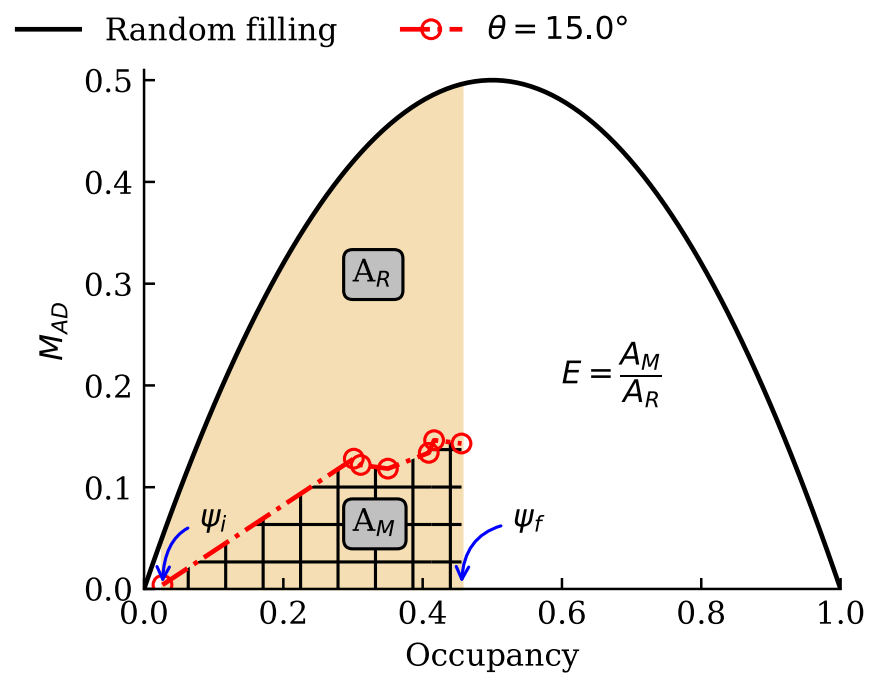

FIG. 1. The mean absolute discrepancy, $M_{A D}$, in filling state for the water-wet case. The black line represents the state in which pores and throats are occupied in a completely random way in the model, Eq. (6). The red line represents the discrepancy in filling state between the model and experiment, Eq. (4). The ratio of the areas shown, $A_{M}$ and $A_{R}$, defined by Eqs. (5) and (7), respectively, is used to define the error measure $E$, Eq. (8).

To quantitatively assess the predictive capability of the model we require a reference simulation; this is the state in which all the pores and throats are filled in a completely random way. If we fill a fraction, $\psi$, of pores and throats with water and $1-\psi$ with oil in a manner that has no systematic correlation with the experiments, then we expect, for a large system, that the mean absolute discrepancy is

$$
M_{A D}^{R}(\psi)=2 \psi(1-\psi) .
$$

This expression takes account of the fact that if the whole rock is filled completely with either oil or water then any model will be correct. The discrepancy only arises during displacement when the location of water-filled elements is incorrect. As before we can define an integral measure, to assess the discrepancy throughout the displacement:

$$
A_{R}(\psi)=\int_{\psi_{i}}^{\psi} M_{A D}^{R}\left(\psi^{\prime}\right) d \psi^{\prime}=\psi^{2}-\frac{2}{3} \psi^{3}-\psi_{i}^{2}+\frac{2}{3} \psi_{i}^{3} .
$$

In the experiments the fluid distribution on a pore-by-pore basis is available for a number of water fractional flows, each one corresponding to a specific occupancy and saturation. These are the experimental points for which we calculate the discrepancy against the model.

We propose an additional quantification of the mismatch, $E$ :

$$
E=\frac{A_{M}\left(\psi_{f}\right)}{A_{R}\left(\psi_{f}\right)} .
$$

This calculation is represented in Fig. 1 for occupancy for the water-wet case. Note that $E=1$ is no better than a random assignment, while $E=0$ is a perfect match. 


\section{Filling order in Expt. Filling order in Model}

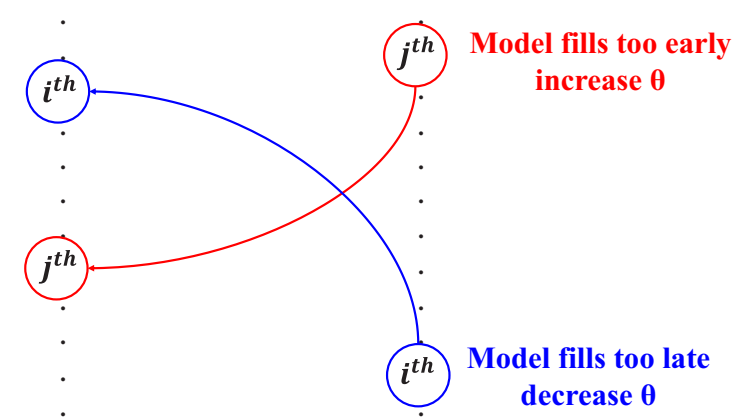

(a)

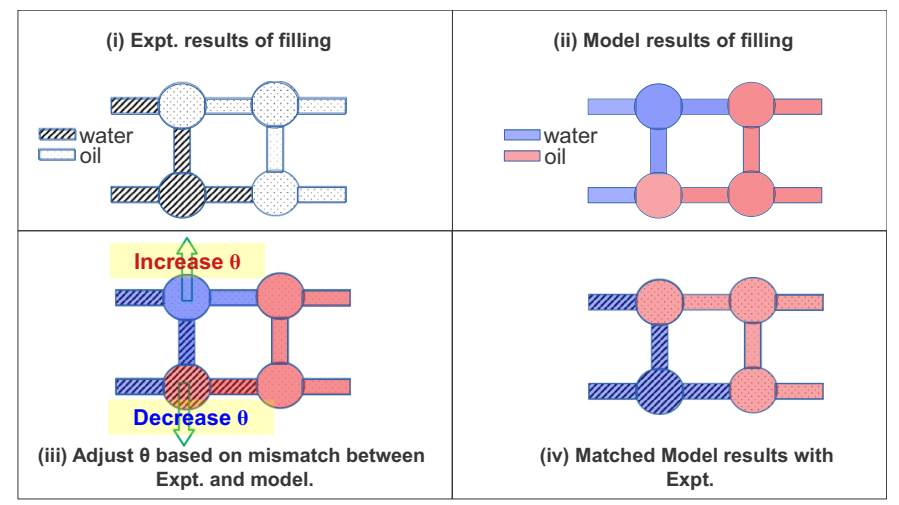

(b)

FIG. 2. (a) A schematic representation of how contact angle is adjusted to obtain correct the filling order. (b) Schematic of the proposed algorithm to remove the mismatch between model and experiment by optimizing the contact angle.

\section{B. Optimization algorithm to minimize pore-by-pore error}

In the pore network model, pores and throats are filled strictly in order of entry capillary pressure assuming quasistatic conditions: in waterflooding those elements that can be filled with the highest entry pressure are filled first and so on in decreasing order of capillary pressure. Hence, if the calculated capillary entry pressures for the pores and throats are not correct then the filling sequence of the model would be different from that in the experiment.

The capillary pressure is a function of wettability and geometry. Hence, if we want to change the capillary entry pressure for each element, we need to change a parameter (wettability or geometry) to obtain the correct filling sequence. As mentioned previously, we do not adjust the static or geometric network properties in this paper.
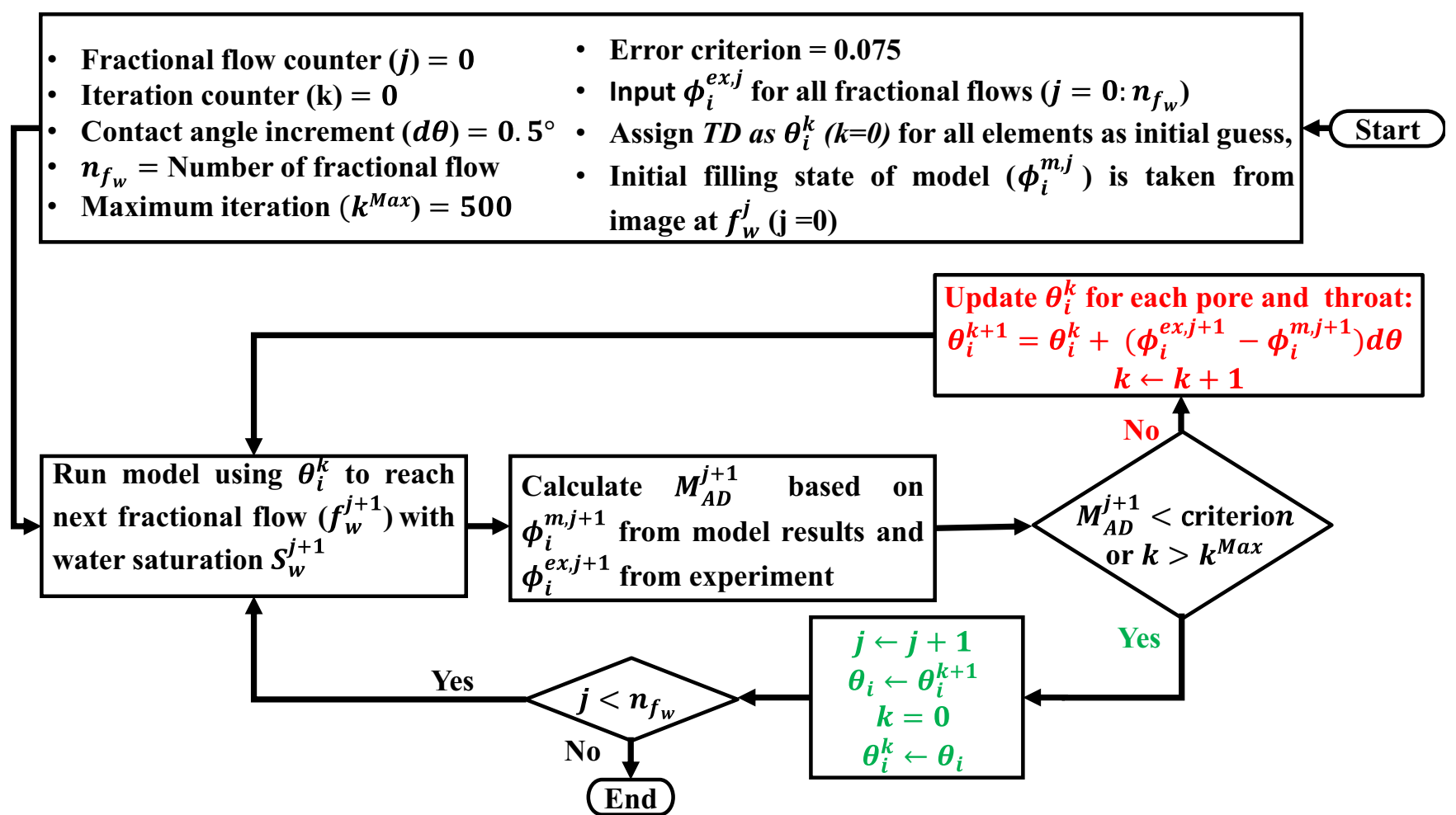

FIG. 3. Calibration algorithm, which optimizes contact angle on a pore-by-pore basis to minimize the error in filling order. Initially the filling state of the elements in the model is taken from the mapped distribution from the experiment at zero fractional flow. Then we run the model to the next fractional flow $\left(f_{w}^{j+1}\right)$ with a water saturation $S_{w}^{j+1}$. At this fractional flow, by comparing the model to the mapped experiment we calculate $M_{A D}^{j+1}$, Eq. (4). Then we compare $M_{A D}^{j+1}$ with our error criterion: if $M_{A D}^{j+1}$ is greater, we need to update the contact angles, and perform another iteration with the updated contact angles, until $M_{A D}^{j+1}$ becomes less than our required error. We then proceed to the next fractional flow until the final fractional flow of 1 . TD is the thermodynamic contact angle. 


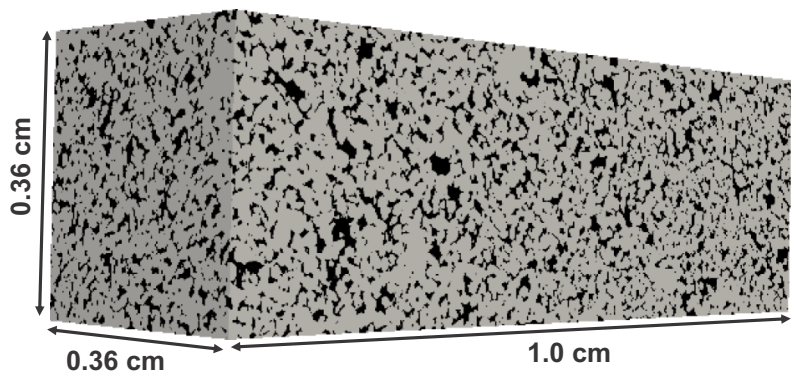

(a)

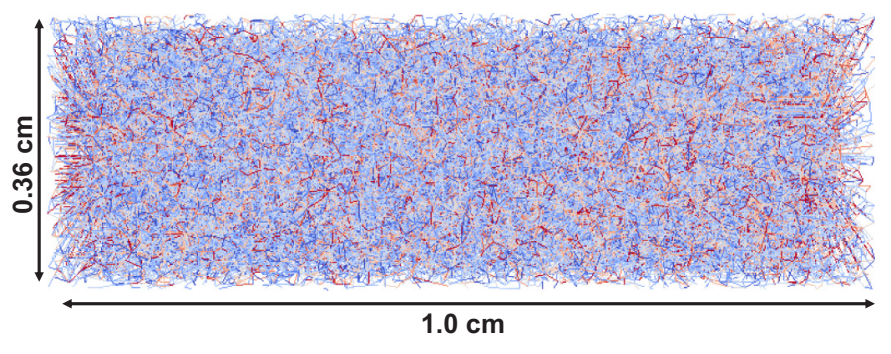

(b)

FIG. 4. (a) The image of dry scan of Bentheimer sandstone used in the water-wet experiment. (b) The structure of the extracted pore network.

Microscopic imaging provides detailed information about the spatial distribution of fluids, but it does not measure the local wetting properties or the surface energy between fluids and solid. Hence, in a model, we need to adjust the surface energies, captured by the contact angle, in such a way that the model accurately predicts the observed fluid distribution.

We will calibrate the wettability by using the contact angle which is assigned to each pore and throat in the network model. However, we do need to have an initial estimate of the contact angles, which, as mentioned above, is the thermodynamic angle. However, it is evident from previous work [41] that there is a wide spatial variation of contact angle that has to be captured to match experimental results accurately while applying uniformly one single value throughout the network will not be sufficient.

As a result, we take the thermodynamic contact angle, deduced from the images, as an initial guess for the contact angle for all pores and throats. We then adjust the values locally, as illustrated in Fig. 2: if an element is filled earlier in the model than in the experiment, the contact angle is increased; in contrast, if an element is filled too late in the model, then the contact angle is decreased.

Based on this idea an algorithm was developed which is based on modifying contact angle for each element to obtain the correct pore filling order. The flowchart for the proposed algorithm is shown in Fig. 3. We start, as input, with the fluid distributions at zero fractional flow of water as the initial condition and perform a simulation in the model until we reach the next largest saturation, which corresponds to the next higher fractional flow. We then compare the mapped experimental distribution with the modeling results and adjust the contact angles as presented in the flowchart in Fig. 3, until we reach the final fractional flow of 1 . When the discrepancy of the modeling results for a given fractional flow becomes less than the predefined error criterion, we proceed to the next higher fractional flow. Note that in each step we have a fixed initial state (corresponding to first fractional flow) and an intermediate state: we run iteratively from the initial state to the intermediate state until the discrepancy is less than some predefined criterion, and then we continue to the next step (the next fractional flow).

The filling state of element $i$ for fractional flow $j$ is $\phi_{i}^{e x, j}$ where $e x$ indicates the experiment. In each iteration, $k$, the contact angle of network elements with the wrong filling state is updated by an increment, $d \theta$, which we choose to be $0.5^{\circ}$.
The new estimate of contact angle is then

$$
\theta_{i}^{k+1}=\theta_{i}^{k}+\left(\phi_{i}^{e x, j+1}-\phi_{i}^{m, j+1}\right) d \theta .
$$

This algorithm can be classified as an array of gradient descent optimizations. The direction of gradient is obtained heuristically by assuming a monotonic relationship between capillary entry pressure (and hence filling and occupancy) and contact angle. We use Eq. (9) to solve this nonlinear optimization problem. Note that gradient descent is a first-order iterative optimization algorithm for finding a local minimum of a function [46]. For this inverse problem we have used a physically based approach, adjusting the contact angle as a proxy for changing the filling order during a displacement. As we show in the Results, this does result in a stable, robust, and simple method to obtain accurate predictions.

$\theta_{i}^{k}$ is initialized with the thermodynamic contact angle (TD). In each iteration, $k$, the network model performs a complete waterflood simulation and $M_{A D}^{j+1}$ is evaluated. If $M_{A D}^{j+1}$ is less than the minimum error or $k$ is larger than predefined maximum iteration number, $k^{\text {Max }}$, the contact angle updating loop will be terminated and we proceed to the next fractional flow; otherwise we continue updating. Here $k^{\operatorname{Max}}$ is selected to be 500 and the error criterion is 0.075 ; that is we stop iterating when $M_{A D}$, Eq. (4), is less than 0.075 .

The choice of error criterion was driven by a number of considerations. Firstly, particularly in smaller elements, there are ambiguities in the segmentation of the image which makes it impossible for a model to match the occupancy perfectly. In addition to this, we have some phase rearrangement especially for the mixed-wet cases. In other words, during waterflooding we observe some elements change to oil-filled from waterfilled. This effect, the local displacement of water by oil in a process where overall water displaces oil, cannot be captured in our model. Another thing that should be taken into consideration is the effect of matching discrepancy in later fractional flows on the earlier match: it is possible to alter the filling sequence earlier in the displacement, which can lead to a deterioration in the overall accuracy of the model.

As mentioned previously, Andrew et al. [26,27] performed a series of repeated drainage and imbibition experiments for brine- $\mathrm{CO}_{2}$ flooding. Later Raeini et al. [28] analyzed this data set and evaluated $M_{A D}$, Eq. (4), between pairs of experimental data, at the end of imbibition experiments. Based on this analysis; for the Bentheimer (the same rock as studied in this 
paper) water-wet sample $M_{A D}$ varied in the range 5.6-13.2\% and for the Ketton water-wet sample the range was 11.2$17.7 \%$ [28]. Hence in our optimization routine we attempt to reduce the mismatch to that at the lower end of observations on repeat experiments in the same sample.

Based on the above discussion, it should be mentioned that the $M_{A D}$ could not be decreased to zero. However, how much we can decrease discrepancy is different from one sample to another. If we try to over-tune the filling states in each fractional flow, it affects the earlier filling order. So, we selected a nonzero error criterion $(0.075)$ which provided a good match to the observed filling state throughout the displacement.

We assessed the convergence and stability of our optimization algorithm by adjusting the maximum number of iterations and the error criterion. Using an error criterion of 0.05 or 0.1 did not significantly improve the results, in that the overall error $(E)$ remained the same to within 1 part in 7 , while the difference in the predicted macroscopic properties, relative permeability, and capillary pressure, was less than $2.9 \%$ on average. Increasing the maximum number of iterations also had an insignificant effect on the results. In all cases during the iteration, the error either decreased or remained constant: we did not observe any instability in the optimization.

The pseudocode for this algorithm is presented in Algorithm 1.

Algorithm 1. Pseudocode for the optimization algorithm

$$
\begin{array}{cl}
\text { Input: } \quad \text { In addition to inputs for model } \\
& n_{f_{w}} \leftarrow \text { number of fractional flow } \\
& \text { Input } \phi_{i}^{e x, j} \text { for all fractional flows } \\
& \left(j=0: n_{f_{w}}\right) \\
& \text { Error criterion } \leftarrow 0.075 \\
& j \leftarrow 0 \\
& k \leftarrow 0 \\
& d \theta \leftarrow 0.5^{\circ} \\
& \theta_{i}^{k} \leftarrow T D \\
& \text { Maximum iteration }\left(k^{M a x}\right) \leftarrow 500 \\
& \phi_{i}^{m, j} \text { for } j=0 \text { is taken from mapped image } \\
& \text { at } f_{w}^{j}(j=0) \\
& \theta_{i} \\
\text { Output: } & \\
\text { While } j<n_{f_{w}} \text { do } \\
M_{A D}^{j+1} \leftarrow 1 \\
\text { While } M_{A D}^{j+1}>C r i t e r i o n ~ \& k \leqslant k^{M a x} \text { do } \\
\quad \text { Run model to } S_{w}^{j+1} \text { correspond to } f_{w}^{j+1} ; \\
\text { Export } \phi_{i}^{m, j+1} \text { from model; } \\
\text { Calculate } M_{A D}^{j+1} \text { based on } \phi_{i}^{m, j+1} \text { and } \phi_{i}^{e x, j+1} ; \\
\text { Update contact angle as follows: } \\
\theta_{i}^{k+1} \leftarrow \theta_{i}^{k}+\left(\phi_{i}^{m, j+1}-\phi_{i}^{e x, j+1}\right) d \theta ; \\
k \leftarrow k+1 ; \\
\text { end } \\
\theta_{i} \leftarrow \theta_{i}^{k} ; \\
k \leftarrow 0 ; \\
\theta_{i}^{k} \leftarrow \theta_{i} ; \\
j \leftarrow & j+1 ; \\
\text { end } \quad &
\end{array}
$$

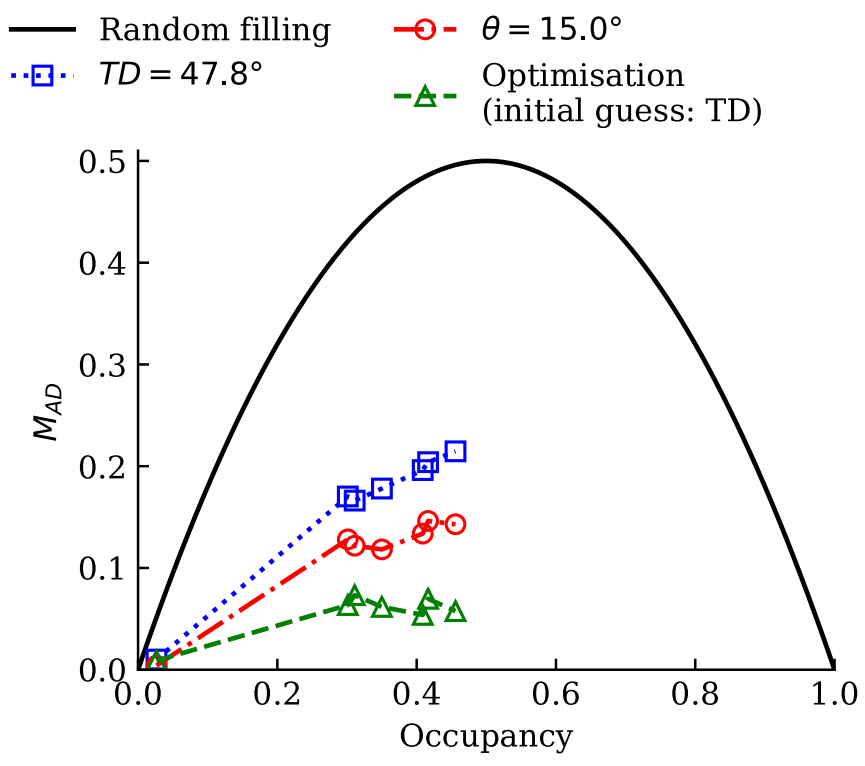

FIG. 5. $M_{A D}$, Eq. (4), as a function of occupancy comparing the discrepancy between experiment and the model for constant contact angle, average thermodynamic contact angle, TD, and the distribution of contact angle from the optimization algorithm.

\section{RESULTS AND DISCUSSION}

\section{A. Water-wet case}

Using a high-quality dry image before the experiment, the pore network was extracted as described in Sec. II B. The dry scan and extracted pore network is presented in Figs. 4(a) and 4(b), respectively. See Table I for a listing of the image size and number of extracted pores and throats.

\section{Pore-by-pore comparison}

The results for pore-by-pore discrepancy in the filling state are presented in Fig. 5. These include model simulations for uniform contact angle, the thermodynamic contact angle as a single average value, and the distribution of contact angle for the optimization algorithm developed in this study. An average of $15^{\circ}$ represents the constant value that provides the lowest mismatch to the experiment. As can be seen, the optimization algorithm was able to reduce the discrepancy at the end of the waterflooding, $M_{A D}$ in Eq. (4), to a value of 0.058 , which is as small as the lowest uncertainty in repeated experiments [26-28]. In addition, the value of error, $E$ in Eq. (8), is equal to 0.14 .

Figure 6 is another way to present the quality of the match for the optimized pore network model. This figure displays the size distribution of the water-filled pores and throats in the experiment and the model after waterflood. It also indicates the pores and throats which are filled with a different fluid in the model compared to the experiment, following the analysis performed previously $[21,31,32]$. It should be noted that the area under the curve, representing wrongly filled pores and throats, provides a graphical representation of the discrepancy. We correctly place the wetting phase, water, in the smaller elements, and oil, the nonwetting phase, in the larger pores and throats, as expected. The discrepancies are in elements 


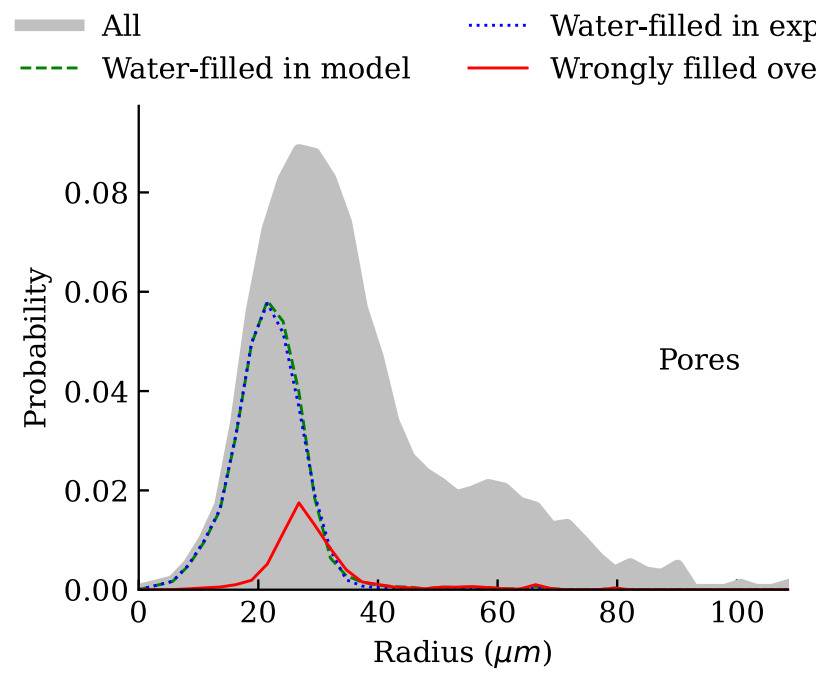

(a)

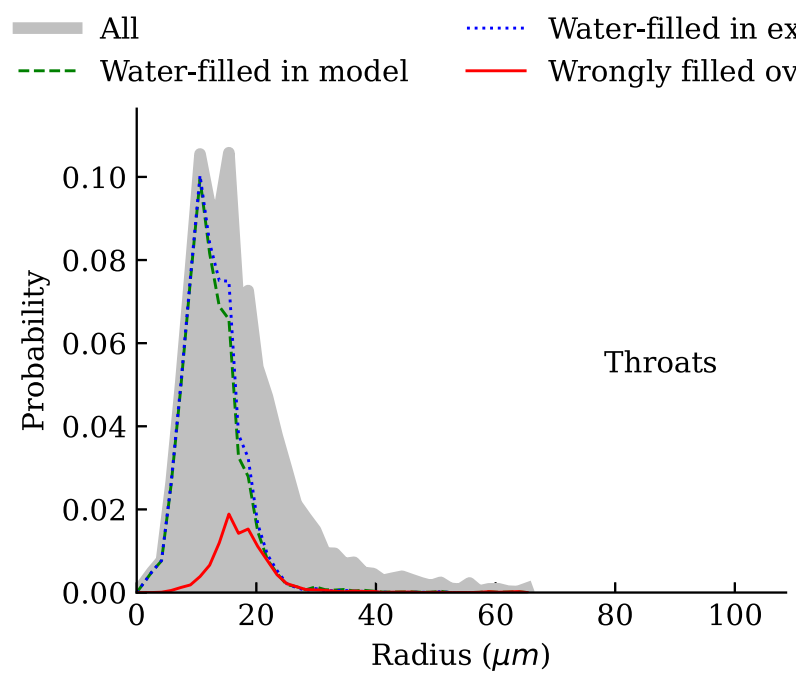

(b)

FIG. 6. Pore and throat sizes that are filled with water at the end of waterflooding for the optimized contact angles (water-wet case).

of intermediate size, some of which are water-filled in the experiment and some oil-filled.

Figure 7 shows the distribution of contact angle obtained for the water-wet sample. As can be seen, the contact angles in $47.1 \%$ of the pores and $53.9 \%$ of the throats remain unchanged. For the remaining pores and throats, using the optimization algorithm results in a spread of contact angle values around the average, where $87.2 \%$ of these pores and $89.2 \%$ of throats have a contact angle difference of less than $15^{\circ}$ compared to the thermodynamic value.

For comparison, the directly measured angle distribution for the water-wet system has a mean value of $66.4^{\circ}$ with a standard deviation of $15.1^{\circ}$ [43]. As discussed before, the geometric angle, in the water-wet case, does tend to overestimate the value in a displacement: our optimized case has a mean contact angle of $47.1^{\circ}$ and a standard deviation of $9.1^{\circ}$, giving a tighter range of smaller values around the average thermodynamic value, as expected for this rock $[39,43]$.

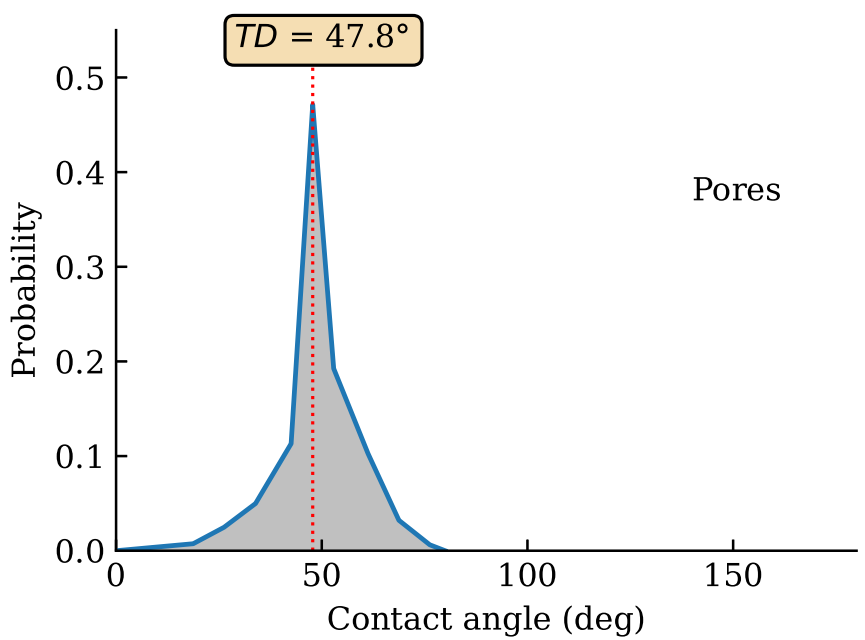

(a)

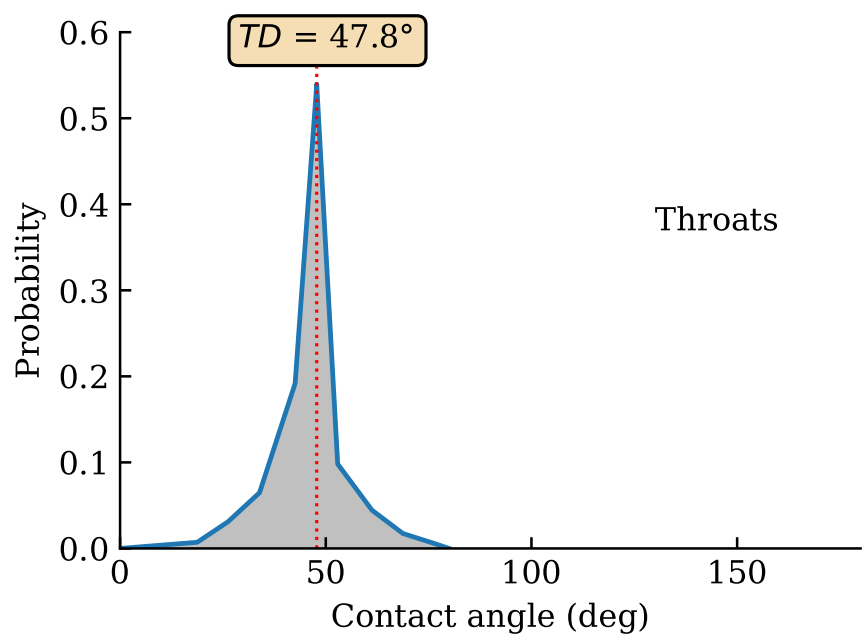

(b)

FIG. 7. Optimized contact angle for (a) pores and (b) throats for the water-wet sample. The thermodynamic contact angle of $47.8^{\circ}$ was used as the initial guess. For both pores and throats, approximately half of the optimized contact angles are unchanged.

\section{Prediction of capillary pressure and relative permeability}

Furthermore, we compare predicted macroscopic properties to the reported experimental data, starting with capillary pressure. In the experiment the capillary pressure was measured from the curvature of the oil-water interfaces using the Young-Laplace equation and the measured interfacial tension [43]. The capillary pressure in the model is computed from the displacement pressures. To assess the importance of initial guess for contact angle we present results for three initial contact angles. The reported TD contact angle for this sample is $47.8^{\circ}$ [39]. Here, we used the TD contact angle, $10^{\circ}$ lower, and $10^{\circ}$ higher than the TD angle as the initial guess. As shown in Fig. 8, the TD contact angle as the initial guess for optimization leads to a good agreement for capillary pressure, while lower or higher initial guess results in a deviation from the experimental results since all the values are artificially shifted higher or lower, while preserving the same rank order 


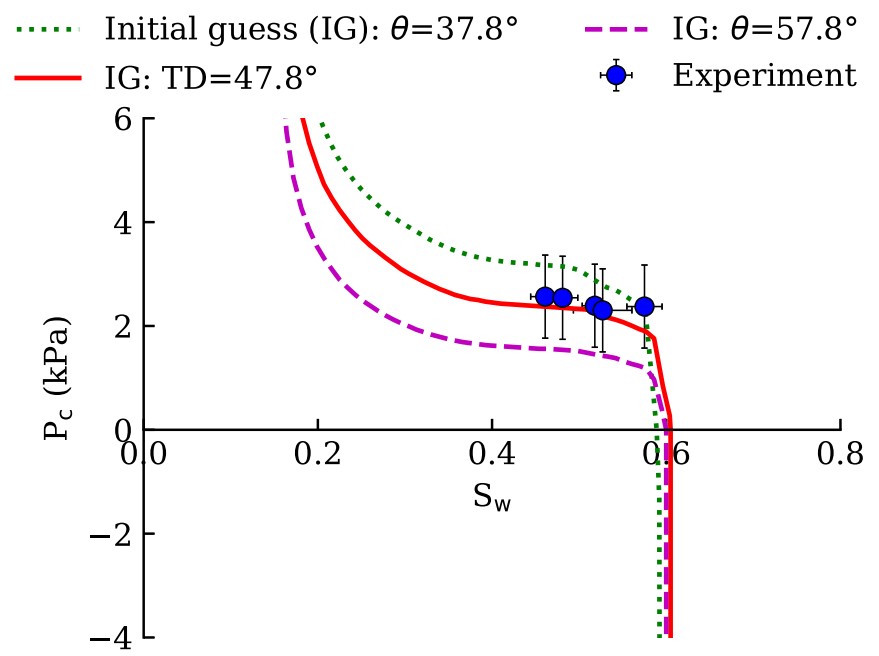

FIG. 8. Capillary pressure based on our optimization algorithm with the three initial conditions. The error bars represent the uncertainties in the experimental measurements.

to honor the filling sequence. Overall we are able to predict the contact angle to within the uncertainties of the measurement using a physically based initial guess of the contact angle. The advantage of the model is that it can then predict the capillary pressure for the full saturation range and, if needed, for other displacement cycles, such as secondary drainage.

Next, the relative permeability based on the optimized contact angle distribution was predicted and compared against the experimental results in Fig. 9. As can be seen, the relative permeability results are also in agreement with experimental data within the uncertainties in the measurements. For comparison, results of other relative permeability measurements on Bentheimer sandstone, which have been performed in the literature, are also presented [47-50], which again show a good agreement with the model predictions within the scatter of the data.

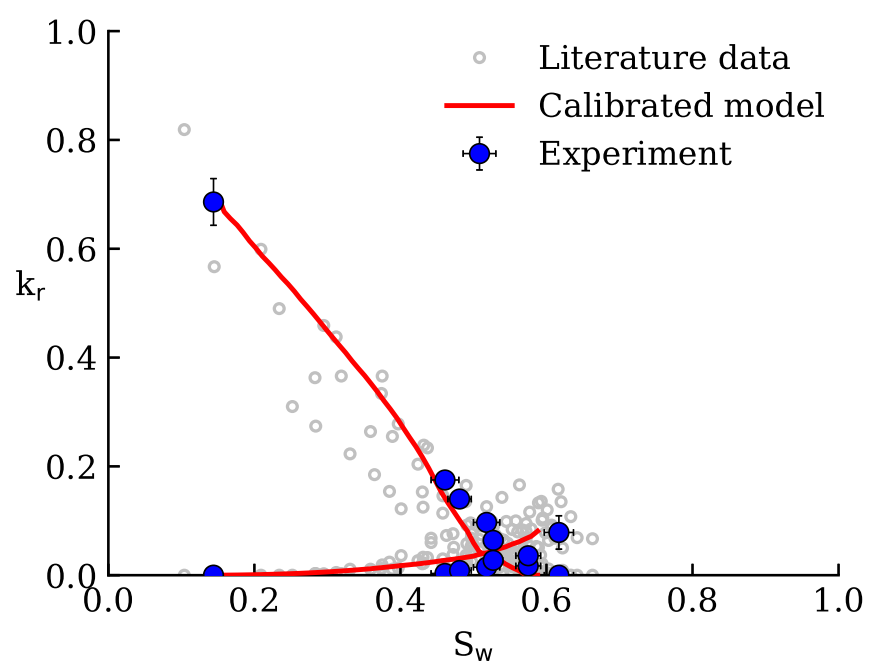

FIG. 9. Relative permeability results based on optimized contact angle distribution. For comparison, other experimental data on waterwet Bentheimer sandstone rocks in the literature [47-50] are shown.

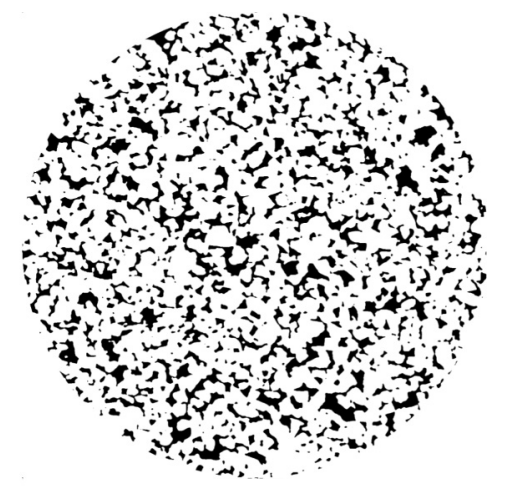

(a)

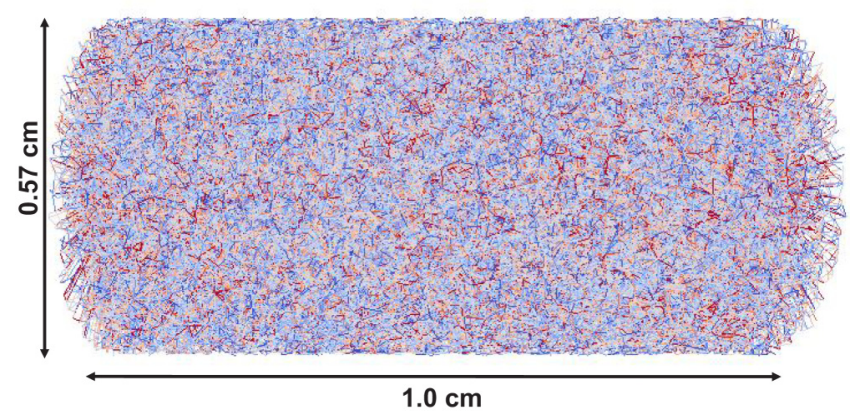

(b)

FIG. 10. (a) A two-dimensional cross section of the threedimensional dry scan of Bentheimer sandstone used in the mixed-wet experiment. (b) The structure of the pore network that was extracted.

This analysis indicates that through matching the correct displacement sequence, we can make good predictions of relative permeability and capillary pressure. However, pore-scale models have already been able to provide good predictions of macroscopic properties for water-wet sandstones [17,19,5052]. Next we will consider a more challenging case, a mixedwet sample where hitherto any predictions have required an unconstrained tuning of contact angle to provide fits to the data.

\section{B. Mixed-wet case}

The experiment on the mixed-wet Bentheimer sample was performed at 8 fractional flows from 0 to 1 . The size of dry scan is $1600 \times 1600 \times 3000$, while the cross section of images is circular. A cross section of the sample and the extracted network are displayed in Fig. 10: see Table I for a listing of the image size and number of extracted pores and throats.

\section{Pore-by-pore comparison}

Figure 11 shows the results for discrepancy for different modeling scenarios, including the best uniform contact angle in terms of our error measure, $E$. In this case the thermodynamic contact angle, which is equal to $98^{\circ}$ [39], was used as the initial estimate in the optimization method. When a bestmatched uniform contact angle or the thermodynamic contact angle were used, the model essentially has no predictive capability and the mismatch is similar to the random filling state. 


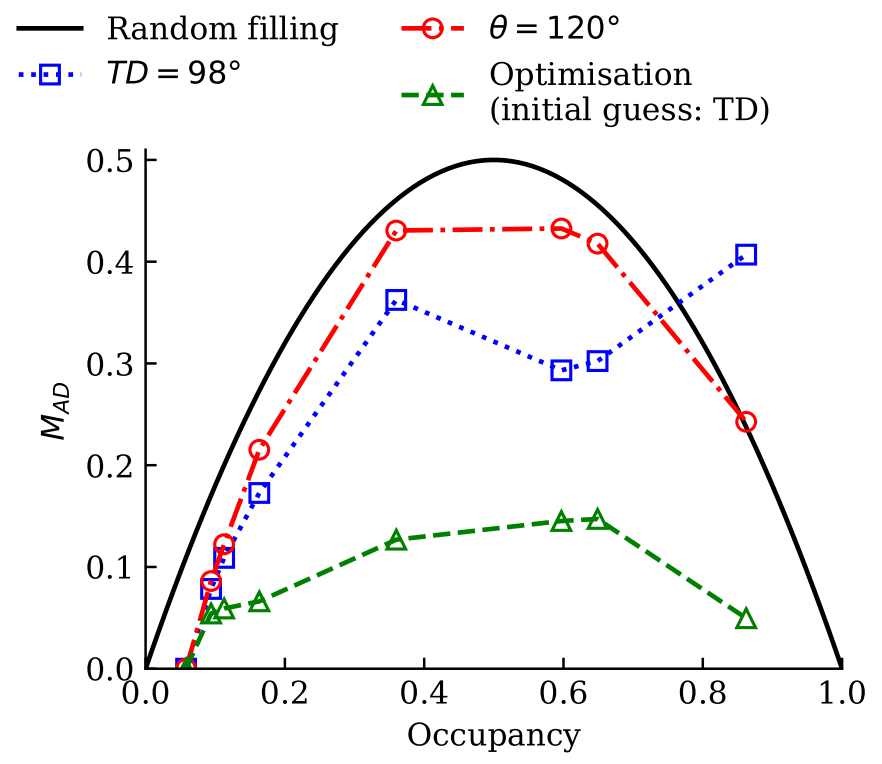

FIG. 11. $M_{A D}$, Eq. (4), as a function of occupancy comparing the discrepancy between experiment and the model for constant contact angle, average thermodynamic contact angle, TD, and the distribution of contact angle from the optimization algorithm.

This behavior can be attributed to the nature of pore filling in the mixed-wet case. Lin et al. [43] showed for this experiment that general assumption of filling pores in order of size is not applicable. Instead, as evident in Fig. 12, we see filling of pores and throats of all size. Hence, if we assume a uniform contact angle we cannot reproduce this behavior: if the contact angle is below $90^{\circ}$ the smaller elements are filled preferentially, whereas for angles above $90^{\circ}$ the larger elements are filled with water first. Alhamadi et al. [53] also observed that for a mixed-wet carbonate during waterflooding, the filling sequence is controlled by both pore size and wettability. What is needed, therefore, is a range of contact angle that allows the filling sequence observed experimentally with both large and small elements filled throughout the displacement.

The optimization workflow leads to a decrease in discrepancy such that we have $M_{A D}=4.3 \%$ at the end point, and $E$ $=0.27$. As seen in Fig. 12, only a small portion of pores and throats are wrongly filled in the model. We cannot eliminate the discrepancy completely, because as discussed in Sec. III B, some water-filled elements are displaced by oil, as also seen in mixed-wet rocks by Rücker et al. [36]. When we try to match the pore occupancy of a given fractional flow it has an effect on the earlier pore and throat filling states to some extent. As a result, through matching $M_{A D}$ at the later fractional flows, the mismatch $M_{A D}$ earlier becomes worse and exceeds the error criterion. However, overall, the error is small and below the uncertainties observed between repeat experiments.

The distribution of contact angle of pores and throats are displayed in Fig. 13. While in the water wet sample, contact angles for almost half of pores and more than half of throats were unchanged, for the mixed-wet case $80.1 \%$ of pore contact angles were changed from the initial guess of the thermodynamic contact angle; for throats $75.6 \%$ were changed. This indicates the significance of needing a range of contact angle to match the data, rather than a single representative
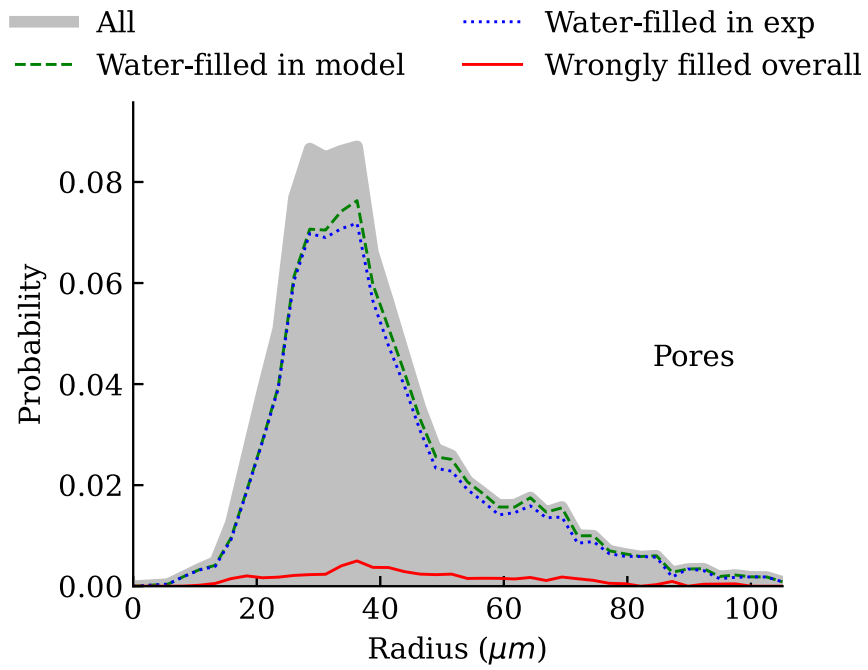

(a)

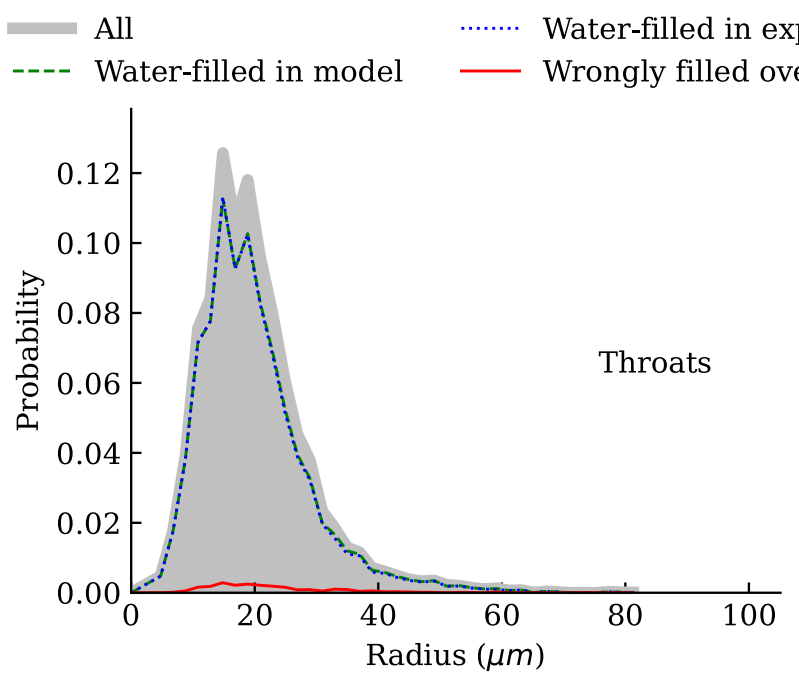

(b)

FIG. 12. Pore and throat sizes that are filled with water at the end of waterflooding for the optimized contact angle distribution (mixedwet case).

value. The measured geometric contact angle distribution in the experiments has a mean value of $80.3^{\circ}$ with a standard deviation of $17.0^{\circ}$ [43]. In this case, as shown previously, the directly measured geometric angle tends to underestimate the value seen during a displacement [39]. Our optimized model predicts a mean of $110.2^{\circ}$ and a standard deviation of $32.1^{\circ}$. In this case we shift the contact angles to values higher than the thermodynamic value with a wide range, needed to capture the filling of elements of all size during the displacement.

\section{Prediction of capillary pressure and relative permeability}

Figure 14 shows capillary pressure obtained from the calibrated pore network model versus capillary pressure obtained by image analysis by Lin et al. [43] for the mixedwet experiment. There is a good agreement between model and experiment, with the model predictions exhibiting higher 


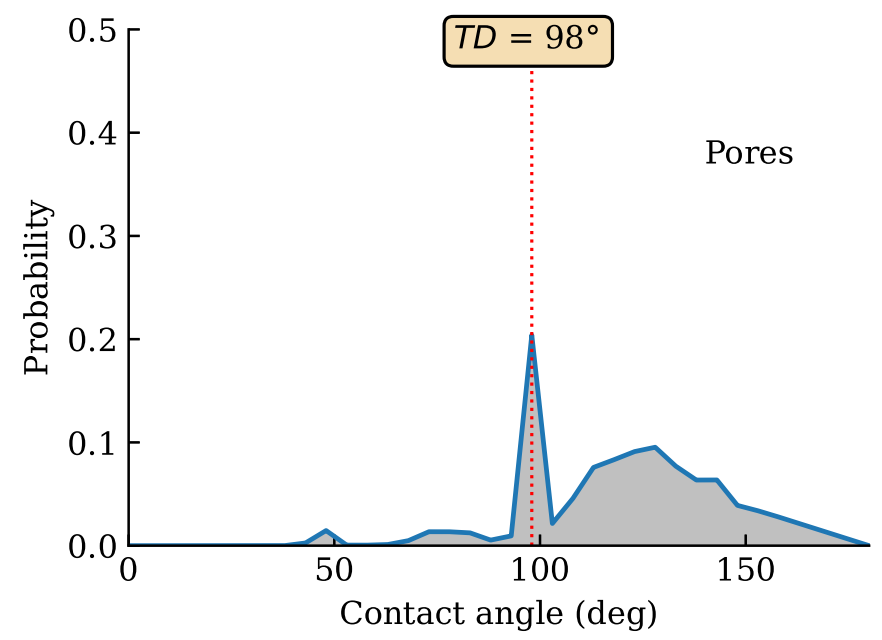

(a)

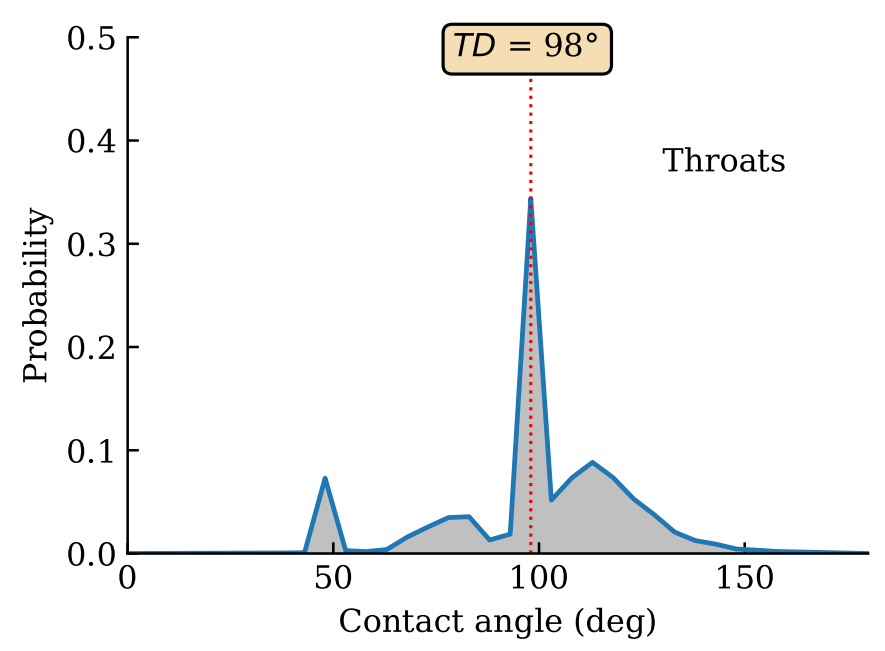

(b)

FIG. 13. Optimized contact angle for (a) pores and (b) throats for the mixed-wet sample. The thermodynamic contact angle of $98^{\circ}$ was used as the initial guess.

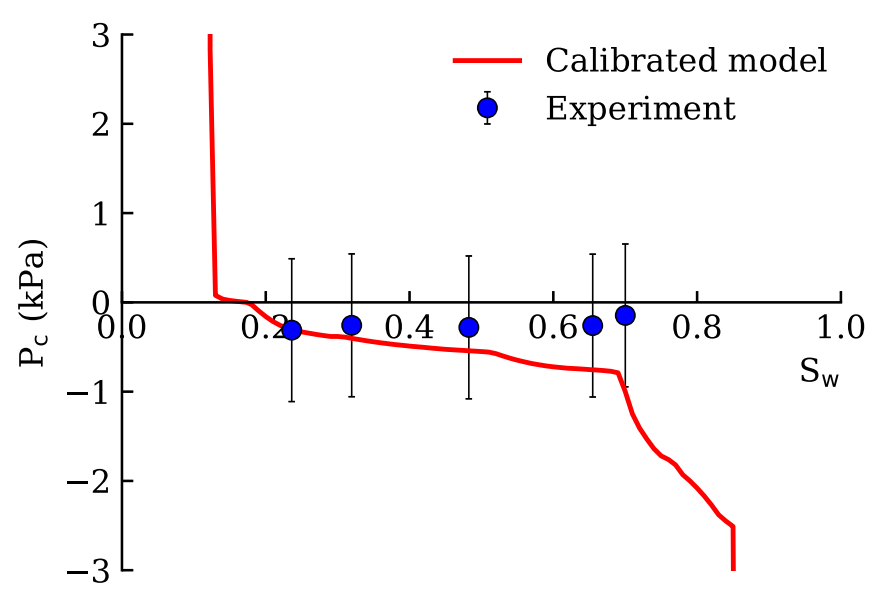

FIG. 14. Capillary pressure obtained from the optimized contact angle distribution for mixed-wet Bentheimer sandstone using the thermodynamic contact angle as the initial guess. The error bars indicate the uncertainties in the experimental measurements.

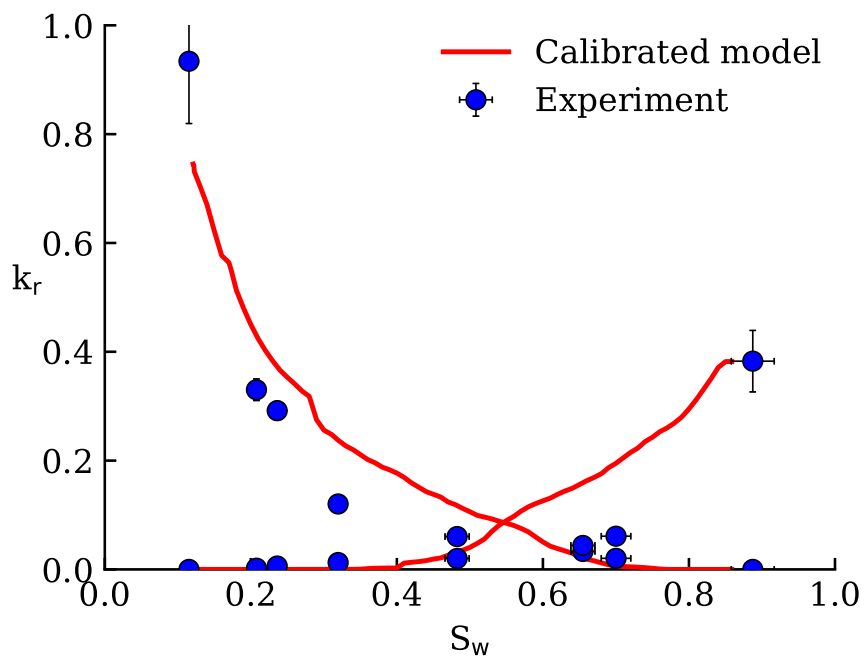

FIG. 15. Relative permeability based on the optimized contact angle distribution. For comparison experimental data on the corresponding mixed-wet Bentheimer sandstone are presented.

absolute values of the capillary pressure near the end points, beyond the range of the experimental data.

The relative permeability of the calibrated network is in good agreement with the experimental data, as shown in Fig. 15. However, at the initial water saturation there is a difference between the oil relative permeability of network model and experimental data. This could be attributed to discrepancies in the assignment of conductivity at the initial condition.

Enhancing image quality could decrease the uncertainty in the imaging and would give us the possibility to determine thermodynamic contact angle on the pore-by-pore basis [39], which could be used as an initial guess to accelerate convergence. Also, high resolution images can give us the opportunity of using saturation instead of filling state in the optimization algorithm. Furthermore, for future work, more sophisticated optimization techniques to solve inverse problems such as Markov chain, data assimilation, and evolutionary'techniques could be tested.

\section{Evidence for contact angle correlation}

In the previous section, it was shown that using the optimization workflow that considers the thermodynamic contact angle as the initial guess, we were able to arrive at a distribution of contact angle which leads to a pore-bypore discrepancy in filling state similar to or less than the differences between repeated experiments. At the same time, the macroscopic properties of $k_{r}$ and $P_{c}$ obtained by this method agreed well with the experimental measurements. In this section, we analyze the contact angle distribution that allowed for a good agreement between model predictions and the experiments for both water-wet and mixed-wet samples.

We start our analysis with an assessment of how the results are changed if we keep the same distribution of contact angle but distribute it randomly in the network: that is, we do not associate a specific angle with a particular element, but simply give an element a value from the optimized distribution. Here, 


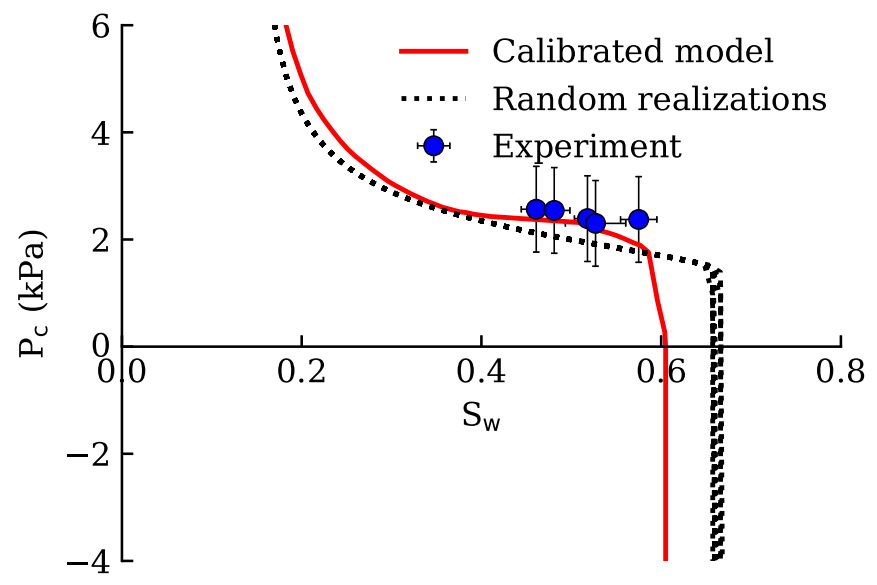

(a)

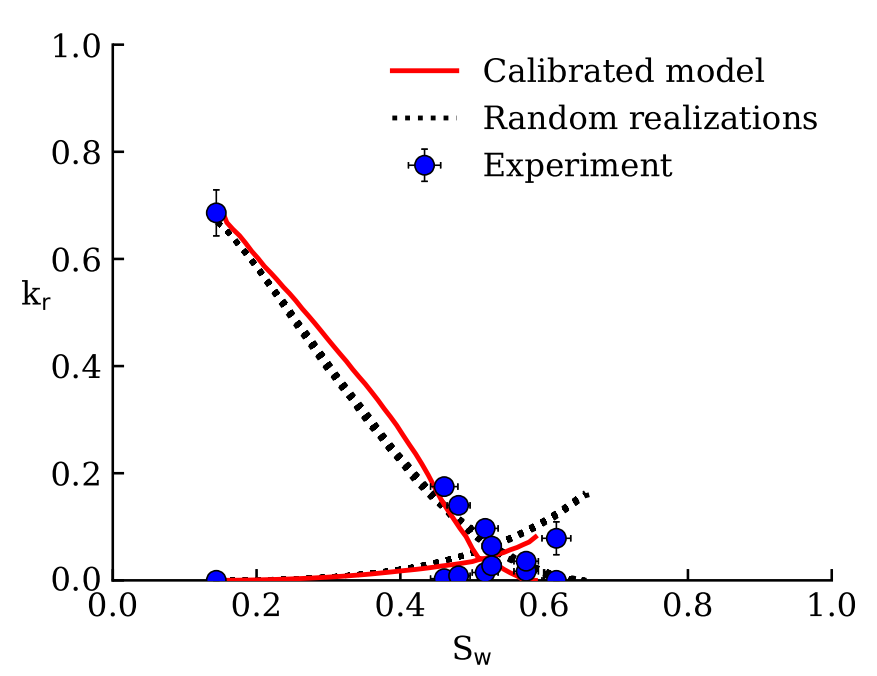

(b)

FIG. 16. (a) Capillary pressure and (b) relative permeability for water-wet sample obtained using optimised distribution of contact angle, and ten randomly redistributed realizations of optimized contact angle.

pore network modeling is performed for ten realizations of redistributed pore and throat contact angles.

The results for capillary pressure and relative permeability for the water-wet case are shown in Fig. 16. The difference between results for the optimized distribution and the randomly redistributed one is seen for higher water saturation where the oil phase loses connectivity. However, it can also be observed that both model predictions for capillary pressure and relative permeability agree to a good extent with experiment.

For the mixed-wet case, Fig. 17, the random contact angle distribution cannot reproduce the experimentally measured macroscopic properties, unlike the optimised model results. With a spatially random distribution, the water relative permeability is underestimated significantly while the residual oil saturation is overestimated. Hence it can be inferred that the contact angle distribution should have some kind of correlation that allows for better connectivity of oil and water near the endpoints.

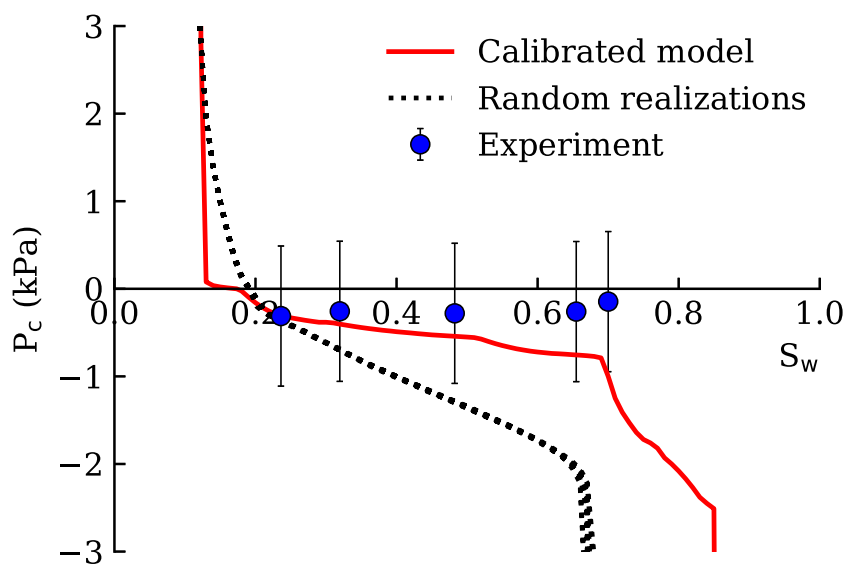

(a)

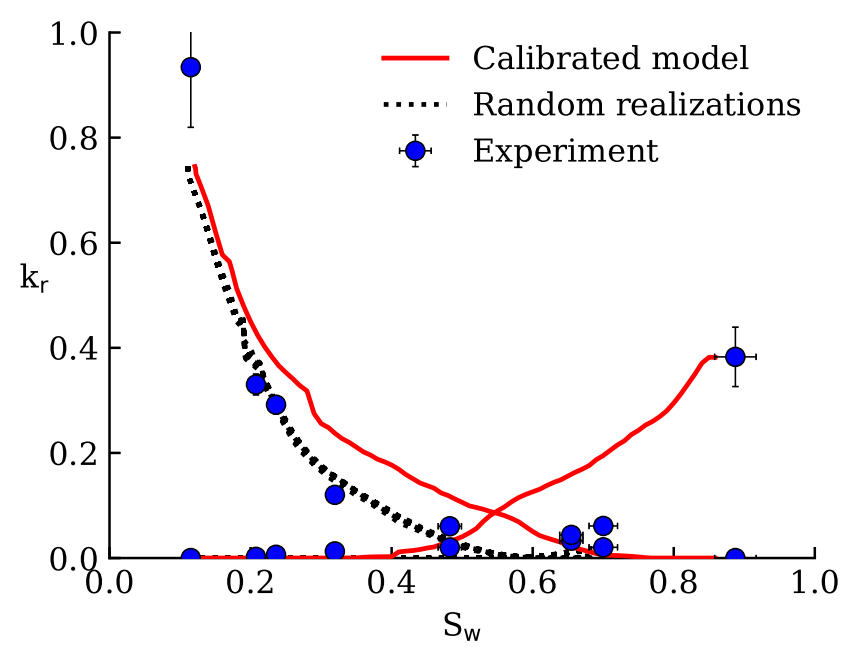

(b)

FIG. 17. (a) Capillary pressure and (b) relative permeability for mixed-wet sample obtained using optimized distribution of contact angle, and ten randomly redistributed realizations of optimised contact angle.

We will study two types of correlation. Firstly, we will investigate if there is a relationship between pore size and wettability: that is, do larger pores and throats tend to be more oil-wet (larger contact angles) or more water-wet. The correlation coefficient between two arbitrary variables $x$ and $y$ denoted by $\rho_{x y}$ is defined as follows:

$$
\rho_{x y}=\frac{\sum_{i}\left(x_{i}-\bar{x}\right)\left(y_{i}-\bar{y}\right)}{\sqrt{\sum_{i}\left(x_{i}-\bar{x}\right)^{2}} \sqrt{\sum_{i}\left(y_{i}-\bar{y}\right)^{2}}} .
$$

Here $x$ is the contact angle for element $i$ while $y$ is the inscribed radius of the element (pore or throat). Note that the sum is over all pores or all throats, so we define correlation coefficients for pores and throats separately. $\bar{x}$ and $\bar{y}$ are average contact angle and average radius.

For the water-wet sample $\rho_{x y}$ for pores is 0.52 and for throats is 0.44 , which means a moderate positive relationship between contact angle of elements and their inscribed radius. For the mixed-wet case $\rho_{x y}$ between contact angle and inscribed radius of pores is 0.70 , which indicates a strong 
positive linear relationship between pore inscribed radius and pore contact angle; similarly for throats $\rho_{x y}$ is equal to 0.68 . Our results are in agreement with previous studies in which the term mixed-wet was used for rocks where the largest pores are oil-wet, with the small pores remaining water-wet [54,55]. This correlation has also been observed directly from contact angle measurements in a mixed-wet reservoir carbonate [40], where again the larger pores tended to be more oil-wet.

Secondly, we will test if the wettability is spatially correlated with clumps of more water-wet and more oilwet pores. The spatial correlation $\xi(d)$ of contact angle from the center of two elements $i$ and $j$ in the network model where $d$ is the Euclidean distance between these two locations

$$
\xi(d)=\frac{\sum_{j} \sum_{i} l_{i j}\left(\theta_{i}-\theta_{j}\right)^{2}}{2 \sigma^{2} \sum_{j} \sum_{i} l_{i j}},
$$

where the sums over $i$ and $j$ are over all pores and all throats. $l_{i j}(d)$ is defined as follows:

$$
l_{i j}(d)= \begin{cases}1 & d-\epsilon<d_{i \rightarrow j}<d+\epsilon \\ 0 & \text { otherwise }\end{cases}
$$

$\sigma^{2}$ is the variance of the contact angle, $d_{i \rightarrow j}$ is the Euclidean distance between centres of the element $i$ and the element $j . \epsilon$ is the statistical length, in this study a length of $2 \mu m$ was used for $\epsilon$. Note that the value of $\xi(d)$ for infinite range or high values of $r$ converges to $1 . \xi(d)=0$ indicates a perfect correlation between two spatially separated values, whereas $\xi(d)=1$ indicates no correlation, and $\xi(d)>1$ represents an anticorrelation.

The spatial correlation for both models is shown in Fig. 18. For comparison, added are the results for spatial correlation in the three reservoir carbonate samples from AlRatrout et al. [56]. These samples include a water-wet sample WW, a mixed-wet sample MW, and an oil-wet sample OW. The correlation length can be regarded as a measure for the stationarity of a specific parameter distribution in space [57]. Here, where $\xi(d)$ becomes stationary is considered the correlation length. Our results indicate the loss of spatial correlation around one average pore diameter for water-wet Bentheimer, which is $45 \mu \mathrm{m}$. On the other hand, the loss of spatial correlation for the mixed-wet sample is around the maximum pore diameter which is $200 \mu \mathrm{m}$ or approximately four times the mean pore diameter. Based on AlRatrout et al. [56] the correlation length for both water-wet and mixed-wet samples were between the minimum and the average pore diameter, while for the oil-wet sample the correlation length was around the maximum pore diameter. In our case, we observe that both samples show some spatial correlation but for the mixed-wet sample the spatial correlation length is higher.

\section{CONCLUSIONS}

Pore network modeling has been used to develop a new method for pore-by-pore comparison of fluid occupancy in multiphase flow. A workflow that optimizes the contact angle distribution was developed and validated against water-wet and mixed-wet steady-state waterflood experiments in Ben-

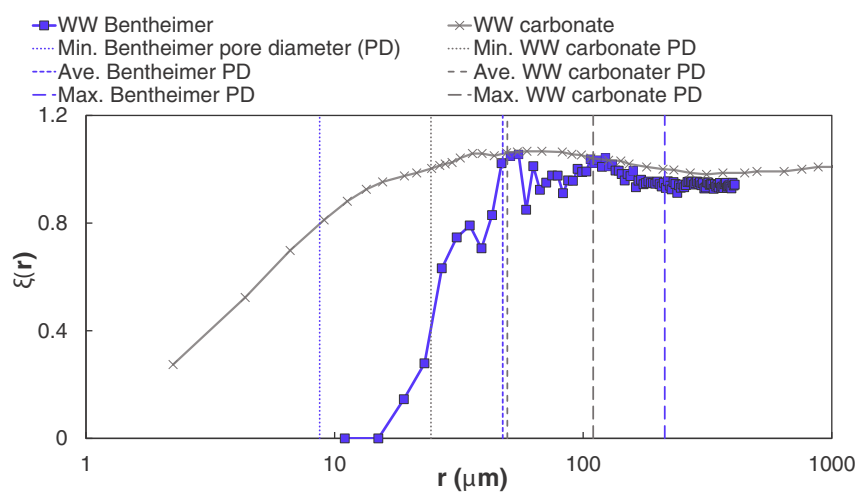

(a)

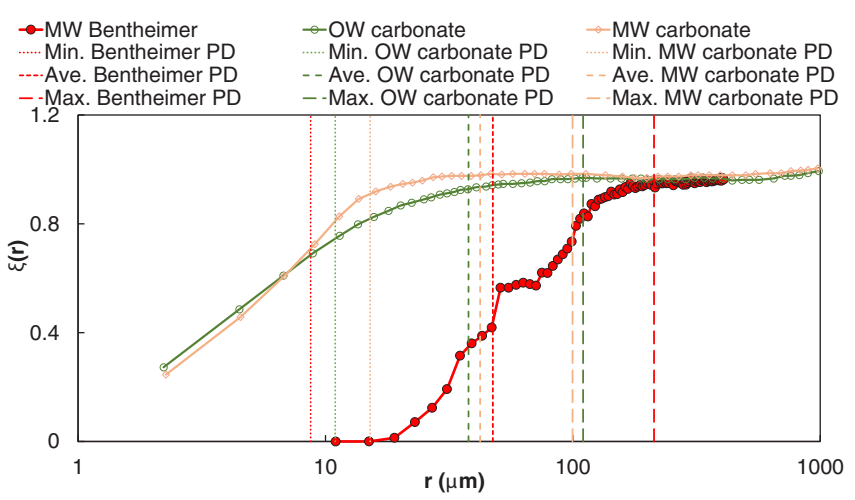

(b)

FIG. 18. Spatial correlation, $\xi(d)$ of (a) water-wet and (b) mixedwet samples. Also, results obtained by AlRatrout et al. [56], for (a) WW carbonate, (b) OW and MW carbonates are presented. Dashed vertical lines are the characteristic lengths representing minimum, mean, and maximum pore diameter size for each sample.

theimer sandstone. The proposed workflow solves a nonlinear inverse problem, based on the adjusting contact angle of each element (pore and throat) to obtain the experimentally observed filling order. The method enabled us to model fluid distribution in the pore space with an uncertainty similar to or lower than repeated experiments.

It has been shown that the initial guess for contact angle has an important effect on the macroscopic parameters. The thermodynamic contact angle obtained from an energy balance applied to the experimental data worked well as an initial guess, but it was necessary to adjust the contact angles of each pore and throat in the model around this base-case value to match the filling sequence observed experimentally.

Once the filling sequence was matched successfully, we predicted capillary pressure and relative permeability as a function of saturation to within the experimental errors in the measurements.

Finally, an analysis of the optimized distribution of contact angle for network elements has shown that there is a mild degree of correlation between pore size and contact angle, with larger pores and throats tending to be more oil-wet. Moreover, we found that there is a spatial correlation in the contact angle distribution; if a spatially random distribution is 
used instead, the predictions of multiphase flow properties are poor, particularly for the mixed-wet sample studied.

In the future, we could extend this study to investigate a wider range of samples, including carbonates and reservoir samples. Furthermore, for steady-state experiments, we only consider a restricted series of fluid configurations. However, unsteady-state experiments, performed with, for instance, fast imaging $[13,15,58]$ may provide a richer assessment of the pore-by-pore displacement dynamics, allowing a more accurate calibration of the network model. Finally, we can then use these calibrated models to predict properties for conditions outside the range studied experimentally.

\section{ACKNOWLEDGMENTS}

We gratefully acknowledge funding from the Shell Digital Rocks program at Imperial College London. We thank S. Berg, S. Masalmeh, J. Freeman, M. Appel, and A. Georgiadis from Shell for helpful and insightful comments on this work.
[1] A. K. Rice, J. E. McCray, and K. Singha, Methane leakage from hydrocarbon wellbores into overlying groundwater: Numerical investigation of the multiphase flow processes governing migration, Water Resour. Res. 54, 2959 (2018).

[2] M. Arshadi, M. Gesho, T. Qin, L. Goual, and M. Piri, Impact of mineralogy and wettability on pore-scale displacement of NAPLs in heterogeneous porous media, J. Contam. Hydrol. 230, 103599 (2020).

[3] I. L. Molnar, J. I. Gerhard, C. S. Willson, and D. M. O'Carroll, Wettability effects on primary drainage mechanisms and NAPL distribution: A pore-scale study, Water Resour. Res. 56, e2019WR025381 (2020).

[4] D. Picchi and I. Battiato, Scaling of two-phase water-steam relative permeability and thermal fluxes in porous media, Int. J. Multiphase Flow 129, 103257 (2020).

[5] Q. Xiong, T. G. Baychev, and A. P. Jivkov, Review of pore network modeling of porous media: Experimental characterisations, network constructions and applications to reactive transport, J. Contam. Hydrol. 192, 101 (2016).

[6] A. G. Hunt and M. Sahimi, Flow, transport, and reaction in porous media: Percolation scaling, critical-path analysis, and effective medium approximation, Rev. Geophys. 55, 993 (2017).

[7] S. Hasan, V. Joekar-Niasar, N. K. Karadimitriou, and M. Sahimi, Saturation dependence of non-Fickian transport in porous media, Water Resour. Res. 55, 1153 (2019).

[8] D. Fadzillah, M. I. Rosli, M. Z. M. Talib, S. K. Kamarudin, and W. R. W. Daud, Review on microstructure modeling of a gas diffusion layer for proton exchange membrane fuel cells, Renew. Sust. Energ. Rev. 77, 1001 (2017).

[9] M. J. Blunt, Multiphase Flow in Permeable Media: A Pore-Scale Perspective (Cambridge University Press, Cambridge, 2017).

[10] K. S. Lari, C. D. Johnston, J. L. Rayner, and G. B. Davis, Field-scale multi-phase LNAPL remediation: Validating a new computational framework against sequential field pilot trials, J. Hazard. Mater. 345, 87 (2018).

[11] Y. Li, G. Blois, F. Kazemifar, and K. T. Christensen, Highspeed quantification of pore-scale multiphase flow of water and supercritical $\mathrm{CO}_{2}$ in 2-d heterogeneous porous micromodels: Flow regimes and interface dynamics, Water Resour. Res. 55, 3758 (2019).

[12] M. Sahimi, Flow and Transport in Porous Media and Fractured Rock: From Classical Methods to Modern Approaches (Wiley, New York, 2011).

[13] D. Wildenschild and A. P. Sheppard, X-ray imaging and analysis techniques for quantifying pore-scale structure and processes in subsurface porous medium systems, Adv. Water Resour. 51, 217 (2013).

[14] M. J. Blunt, B. Bijeljic, H. Dong, O. Gharbi, S. Iglauer, P. Mostaghimi, A. Paluszny, and C. Pentland, Pore-scale imaging and modelling, Adv. Water Resour. 51, 197 (2013).

[15] V. Cnudde and M. N. Boone, High-resolution X-ray computed tomography in geosciences: A review of the current technology and applications, Earth-Sci. Rev. 123, 1 (2013).

[16] D. Silin, L. Tomutsa, S. M. Benson, and T. W. Patzek, Microtomography and pore-scale modeling of two-phase fluid distribution, Transport Porous Med. 86, 495 (2011).

[17] A. Aghaei and M. Piri, Direct pore-to-core up-scaling of displacement processes: Dynamic pore network modeling and experimentation, J. Hydrology 522, 488 (2015).

[18] N. Idowu, C. Nardi, H. Long, P.-E. Øren, and I. Bondino, Improving digital rock physics predictive potential for relative permeabilities from equivalent pore networks, in International Symposium of the Society of Core Analysts (Society of Core Analysts, Napa Valley, California, 2013), pp. SCA2013-017.

[19] P. H. Valvatne and M. J. Blunt, Predictive pore-scale modeling of two-phase flow in mixed wet media, Water Resour. Res. 40, W07406 (2004).

[20] L. Ruspini, R. Farokhpoor, and P. Øren, Pore-scale modeling of capillary trapping in water-wet porous media: A new cooperative pore-body filling model, Adv. Water Resour. 108, 1 (2017).

[21] T. Bultreys, Q. Lin, Y. Gao, A. Q. Raeini, A. AlRatrout, B. Bijeljic, and M. J. Blunt, Validation of model predictions of pore-scale fluid distributions during two-phase flow, Phys. Rev. E 97, 053104 (2018).

[22] I. Bondino, G. Hamon, W. Kallel, D. Kac et al., Relative permeabilities from simulation in $3 \mathrm{~d}$ rock models and equivalent pore networks: Critical review and way forward, Petrophysics 54, 538 (2013).

[23] A. Skauge, K. Spildo, L. Høiland, and B. Vik, Theoretical and experimental evidence of different wettability classes, J. Pet. Sci. Eng. 57, 321 (2007).

[24] P. Meakin and A. M. Tartakovsky, Modeling and simulation of pore-scale multiphase fluid flow and reactive transport in fractured and porous media, Rev. Geophys. 47, RG3002 (2009).

[25] C. A. Reynolds, M. J. Blunt, and S. Krevor, Multiphase flow characteristics of heterogeneous rocks from $\mathrm{CO}_{2}$ storage reservoirs in the United Kingdom, Water Resour. Res. 54, 729 (2018).

[26] M. Andrew, B. Bijeljic, and M. J. Blunt, Pore-scale imaging of trapped supercritical carbon dioxide in sandstones and carbonates, Inte. J. Greenhouse Gas Control 22, 1 (2014). 
[27] M. Andrew, B. Bijeljic, and M. J. Blunt, Pore-scale imaging of geological carbon dioxide storage under in situ conditions, Geophys. Res. Lett. 40, 3915 (2013).

[28] A. Q. Raeini, J. Yang, I. Bondino, T. Bultreys, M. J. Blunt, and B. Bijeljic, Validating the generalized pore network model using micro-ct images of two-phase flow, Trans. Porous Med. 130, 405 (2019).

[29] C. Sun, J. E. McClure, P. Mostaghimi, A. L. Herring, M. Shabaninejad, S. Berg, and R. T. Armstrong, Linking continuum-scale state of wetting to pore-scale contact angles in porous media, J. Colloid Interface Sci. 561, 173 (2020).

[30] T. Akai, A. M. Alhammadi, M. J. Blunt, and B. Bijeljic, Modeling oil recovery in mixed-wet rocks: Pore-scale comparison between experiment and simulation, Trans. Porous Med. 127, 393 (2019).

[31] P. Øren, L. Ruspini, M. Saadatfar, R. Sok, M. Knackstedt, and A. Herring, In situ pore-scale imaging and image-based modeling of capillary trapping for geological storage of $\mathrm{CO}_{2}$, Int. J. Greenh. Gas Con. 87, 34 (2019).

[32] T. Bultreys, K. Singh, A. Q. Raeini, L. C. Ruspini, P.-E. Øren, S. Berg, M. Rücker, B. Bijeljic, and M. J. Blunt, Verifying pore network models of imbibition in rocks using time-resolved synchrotron imaging, Water Resour. Res. 56, e2019WR026587 (2020).

[33] R. T. Armstrong, J. E. McClure, M. A. Berrill, M. Rücker, S. Schlüter, and S. Berg, Beyond Darcy's law: The role of phase topology and ganglion dynamics for two-fluid flow, Phys. Rev. E 94, 043113 (2016).

[34] F. Alpak, S. Berg, and I. Zacharoudiou, Prediction of fluid topology and relative permeability in imbibition in sandstone rock by direct numerical simulation, Adv. Water Resour. 122, 49 (2018).

[35] A. AlRatrout, A. Q. Raeini, B. Bijeljic, and M. J. Blunt, Automatic measurement of contact angle in pore-space images, Adv. Water Resour. 109, 158 (2017).

[36] M. Rücker, W.-B. Bartels, K. Singh, N. Brussee, A. Coorn, H. Van Der Linde, A. Bonnin, H. Ott, S. Hassanizadeh, M. Blunt et al., The effect of mixed wettability on pore-scale flow regimes based on a flooding experiment in ketton limestone, Geophys. Res. Lett. 46, 3225 (2019).

[37] N. Saxena, A. Hows, R. Hofmann, F. O. Alpak, J. Dietderich, M. Appel, J. Freeman, and H. De Jong, Rock properties from micro-ct images: Digital rock transforms for resolution, pore volume, and field of view, Adv. Water Resour. 134, 103419 (2019).

[38] N. Saxena, A. Hows, R. Hofmann, J. Freeman, and M. Appel, Estimating pore volume of rocks from pore-scale imaging, Trans. Porous Med. 129, 403 (2019).

[39] M. J. Blunt, Q. Lin, T. Akai, and B. Bijeljic, A thermodynamically consistent characterization of wettability in porous media using high-resolution imaging, J. Colloid Interface Sci. 552, 59 (2019).

[40] A. AlRatrout, M. J. Blunt, and B. Bijeljic, Wettability in complex porous materials, the mixed-wet state, and its relationship to surface roughness, Proc. Natl. Acad. Sci. 115, 8901 (2018).

[41] T. Akai, Q. Lin, B. Bijeljic, and M. J. Blunt, Using energy balance to determine pore-scale wettability, J. Colloid Interface Sci. 576, 486 (2020).
[42] Q. Lin, B. Bijeljic, R. Pini, M. J. Blunt, and S. Krevor, Imaging and measurement of pore-scale interfacial curvature to determine capillary pressure simultaneously with relative permeability, Water Resour. Res. 54, 7046 (2018).

[43] Q. Lin, B. Bijeljic, S. Berg, R. Pini, M. J. Blunt, and S. Krevor, Minimal surfaces in porous media: Pore-scale imaging of multiphase flow in an altered-wettability Bentheimer sandstone, Phys. Rev. E 99, 063105 (2019).

[44] A. Q. Raeini, B. Bijeljic, and M. J. Blunt, Generalized network modeling: Network extraction as a coarse-scale discretization of the void space of porous media, Phys. Rev. E 96, 013312 (2017).

[45] H. Dong and M. J. Blunt, Pore-network extraction from microcomputerized-tomography images, Phys. Rev. E 80, 036307 (2009).

[46] S. Boyd, S. P. Boyd, and L. Vandenberghe, Convex Optimization (Cambridge University Press, Cambridge, 2004).

[47] Y. Gao, Q. Lin, B. Bijeljic, and M. J. Blunt, X-ray microtomography of intermittency in multiphase flow at steady state using a differential imaging method, Water Resour. Res. 53, 10274 (2017).

[48] A. Alizadeh, M. Khishvand, M. Ioannidis, and M. Piri, Multiscale experimental study of carbonated water injection: An effective process for mobilization and recovery of trapped oil, Fuel 132, 219 (2014).

[49] C. Reynolds and S. Krevor, Characterizing flow behavior for gas injection: Relative permeability of $\mathrm{CO}_{2}$-brine and $\mathrm{N}_{2}$ water in heterogeneous rocks, Water Resour. Res. 51, 9464 (2015).

[50] P.-E. Øren, S. Bakke, and O. J. Arntzen, Extending predictive capabilities to network models, SPE Journal 3, 324 (1998).

[51] A. Q. Raeini, B. Bijeljic, and M. J. Blunt, Generalized network modeling of capillary-dominated two-phase flow, Phys. Rev. E 97, 023308 (2018).

[52] B. Raeesi and M. Piri, The effects of wettability and trapping on relationships between interfacial area, capillary pressure and saturation in porous media: A pore-scale network modeling approach, J. Hydrology 376, 337 (2009).

[53] A. M. Alhammadi, Y. Gao, T. Akai, M. J. Blunt, and B. Bijeljic, Pore-scale x-ray imaging with measurement of relative permeability, capillary pressure and oil recovery in a mixedwet micro-porous carbonate reservoir rock, Fuel 268, 117018 (2020).

[54] S. R. McDougall and K. S. Sorbie, The impact of wettability on waterflooding: Pore-scale simulation, SPE Reservoir Engineering 10, 208 (1995).

[55] R. A. Salathiel, Oil recovery by surface film drainage in mixedwettability rocks, J. Pet. Technol. 25, 1216 (1973).

[56] A. AlRatrout, M. J. Blunt, and B. Bijeljic, Spatial correlation of contact angle and curvature in pore-space images, Water Resour. Res. 54, 6133 (2018).

[57] G. Franceschetti and D. Riccio, Scattering, Natural Surfaces, and Fractals (Elsevier, Amsterdam, 2006).

[58] S. Berg, H. Ott, S. A. Klapp, A. Schwing, R. Neiteler, N. Brussee, A. Makurat, L. Leu, F. Enzmann, J.-O. Schwarz, M. Kersten, S. Irvine, and M. Stampanoni, Real-time 3d imaging of haines jumps in porous media flow, Proc. Natl. Acad. Sci. 110, 3755 (2013). 